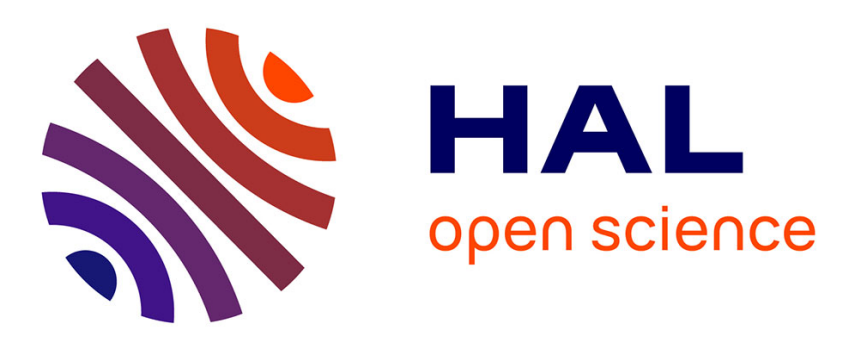

\title{
A Unified Framework for Certificate and Compilation for QBF
}

Igor Stéphan, Benoit da Mota

\section{To cite this version:}

Igor Stéphan, Benoit da Mota. A Unified Framework for Certificate and Compilation for QBF. Third Indian Conference, ICLA 2009, 2009, Chennai, India. pp.210 - 223, 10.1007/978-3-540-92701-3_15 . hal-03255579

\section{HAL Id: hal-03255579 \\ https://univ-angers.hal.science/hal-03255579}

Submitted on 9 Jun 2021

HAL is a multi-disciplinary open access archive for the deposit and dissemination of scientific research documents, whether they are published or not. The documents may come from teaching and research institutions in France or abroad, or from public or private research centers.
L'archive ouverte pluridisciplinaire HAL, est destinée au dépôt et à la diffusion de documents scientifiques de niveau recherche, publiés ou non, émanant des établissements d'enseignement et de recherche français ou étrangers, des laboratoires publics ou privés. 


\title{
A unified framework for Certificate and Compilation for QBF
}

\author{
Igor Stéphan and Benoit Da Mota \\ LERIA University of Angers, France \\ email: \{damota, stephan\}@info.univ-angers.fr
}

\begin{abstract}
We propose in this article a unified framework for certificate and compilation for QBF. We provide a search-based algorithm to compute a certificate for the validity of a QBF and a search-based algorithm to compile a valid $\mathrm{QBF}$ in our unified framework.
\end{abstract}

\section{Introduction}

The quantified Boolean formula $(\mathrm{QBF})$ validity problem is a generalisation of the Boolean formulae satisfiability problem. While the complexity of Boolean satisfiability problem is NP-complete, it is PSPACE-complete for the QBF validity problem. This is the price for a more concise representation of many classes of formulae. Many important problems in several research fields have polynomialtime translations to the QBF validity problem : AI planning [24,1], Bounded Model Construction [1], Formal Verification (see [5] for a survey).

Most of the recent decision procedures for QBF validity [11, 28, 19, 18] are extensions of the search-based Davis-Putnam procedure [15] for Boolean satisfiability. A search-based procedure for QBF chooses one Boolean variable, tries to solve two simpler subproblems and combines the results according to the semantics of the quantifier associated to the variable. Some other decision procedures are based on resolution principle (as Q-resolution [9] which extends the resolution principle for Boolean formulae to QBF or Quantor [6] which combines efficiently Q-resolution and expansion), quantifier-elimination algorithms [23, 22], or skolemization and SAT solvers [2].

Every finite two-player game can be modeled in QBF [17]. In this kind of applications, a decision procedure (the formula is valid or not) is not sufficient since a solution is needed. A solution of a QBF (a QBF model) is a set of Boolean functions [10]. One possibility to represent them is to build a treeshape representation (called policy [13] or strategy [7]) but it is exponential in worst case and unfortunately also in usual ones. With a search-based procedure, it is very easy to build a solution of a QBF from the solutions of its two simpler subproblems $[13,7]$.

When a QBF solver returns valid or non-valid, there is no way to check if the answer is correct while in propositional logic the associated result to the decision (a model) is easy to check. A certificate for a valid QBF is any piece of information that provides self-supporting evidence of validity for that QBF [5]. 
A sat-certificate $[4,3]$ is a representation of a sequence of Boolean functions for a QBF that certifies its validity. This approach seems to us promising since the generated certificate is not linked to the representation of the input QBF but only to its semantics. The computation of a sat-certificate is described in [2] in the framework of sKizzo as a reconstruction from a trace. To the best of our knowledge, there is no result of how to build a sat-certificate of a QBF from the sat-certificates of its two simpler subproblems. It is an important issue since most of the QBF solvers are search-based procedures. Our first contribution is double (Section 4): we define an operator for sat-certificates in order to be able to build a sat-certificate of a QBF from the sat-certificates of its two simpler subproblems and we describe an algorithm which extends any search-based algorithm to build a sat-certificate for a valid QBF during the decision process and not a posteriori from a trace.

In general, a knowledge base is compiled off-line into a target language which is then used on-line to answer some queries. In QBF case, seen as a two-player game, one of the most useful query for the existential player is : what should I play to still be sure to win? Our second contribution is a unified framework for certificate and compilation of QBF (Section 3): the literal base representation which is an extension of the sat-certificate representation. In order to extend any search-based procedure to a QBF compiler, an important issue is how to compute the compilation of a QBF from the result of the compilation of its two simpler subproblems. Our third contribution is also double (Section 5): we define an operator for literal bases in order to be able to compute the compilation of a QBF from the literal bases of its two simpler subproblems and we describe an algorithm which extends any search-based algorithm to compile a valid QBF.

Finally we discusse related work (Section 6) and we draw a conclusion (Section 7).

\section{Preliminaries}

\subsection{Propositional logic.}

The set of propositional variables is denoted by $\mathcal{V}$. Symbols $\perp$ and $T$ are the propositional constants $(\bar{T}=\perp$ and $\bar{\perp}=T)$. Symbol $\wedge$ stands for conjunction, $\vee$ for disjunction, $\neg$ for negation, $\rightarrow$ for implication and $\leftrightarrow$ for bi-implication. A literal is a propositional variable or the negation of a propositional variable. A formula is in conjunctive normal form (CNF) if it is a conjunction of disjunctions of literals. Definitions of the language of propositional formula PROP and semantics of all the Boolean symbols are defined in standard way. A substitution is a function from propositional variables to PROP. This definition is extended as usual to a function from PROP to PROP: $[x \leftarrow F](G)$ is the formula obtained from $G$ by replacing occurrences of the propositional variable $x$ by the formula $F$. This definition is also extended as usual for the substitution of a formula by another formula. An interpretation $v$ is a function from $\mathcal{V}$ to $\{$ true, false ; the extension to PROP is denoted $v^{*}$. To an interpretation $v$ is associated a set in the standard way and a substitution $\boldsymbol{v}$ as follows : if $v(x)=$ true then 
$[x \leftarrow \top]$ is in $\boldsymbol{v}$, if $v(x)=$ false then $[x \leftarrow \perp]$ is in $\boldsymbol{v}$. Propositional satisfaction is denoted $\models\left(v \models F\right.$ means $v^{*}(F)=$ true, the propositional formula $F$ is satisfied by $v$ and $v$ is a model of $F$ ). Logical equivalence is denoted $\equiv$. To a Boolean function $f$ (i.e. a function from $\{\text { true, false }\}^{n}$ to $\{$ true, false $\}$ ) is associated a propositional formula $\psi_{f}$ on the variables $\left\{x_{1}, \ldots, x_{n}\right\}$ such that $v^{*}\left(\psi_{f}\right)=$ true if and only if $f\left(v\left(x_{1}\right), \ldots, v\left(x_{n}\right)\right)=$ true for any interpretation $v$.

\subsection{Quantified Boolean Formulae.}

The symbol $\exists$ stands for the existential quantifier and $\forall$ stands for the universal quantifier ( $q$ stands for any quantifier). A binder $Q$ is a string $q_{1} x_{1} \ldots q_{n} x_{n}$ with $x_{1}, \ldots, x_{n}$ distinct propositional variables and $q_{1} \ldots q_{n}$ quantifiers. It is assumed that distinct quantifiers bind occurrences of distinct propositional variables. The empty string is denoted by $\epsilon$. A (prenex) quantified Boolean formula (QBF) is constituted of a binder and a propositional formula called the matrix (only closed formulae are considered: each variable in the matrix is also in the binder). A QBF is in conjunctive normal form if its matrix is in conjunctive normal form. The semantics of $\mathrm{QBF}$ is defined as follows: for every propositional variable $y$ and every QBF $Q M$

$$
\exists y Q M=(Q[y \leftarrow \top](M) \vee Q[y \leftarrow \perp](M))
$$

and

$$
\forall y Q M=(Q[y \leftarrow \top](M) \wedge Q[y \leftarrow \perp](M)) .
$$

A QBF $F$ is valid if $F \equiv T$. If $y$ is an existentially quantified variable preceded by the universally quantified variables $x_{1}, \ldots, x_{n}$ we denote $\hat{y}_{x_{1} \ldots x_{n}}$ its Skolem function from $\{\text { true, false }\}^{n}$ to $\{$ true, false $\}$. A model for a valid QBF $Q M$ is a sequence $\psi_{\hat{y}_{1}} ; \ldots ; \psi_{\hat{y}_{p}}$ such that $\left[y_{1} \leftarrow \psi_{\hat{y}_{1}}\right] \ldots\left[y_{p} \leftarrow \psi_{\hat{y}_{p}}\right](M)$ is a tautology [10] $\left(y_{1}, \ldots, y_{p}\right.$ the existentially quantified variables of $\left.Q M\right)$. For example, the QBF $\exists a \exists b \forall c((a \vee b) \leftrightarrow c)$ (since $\exists a \exists b \forall c((a \vee b) \leftrightarrow c) \equiv \exists a \exists b(((a \vee b) \leftrightarrow \top) \wedge((a \vee b) \leftrightarrow \perp)) \equiv$ $\perp)$ but the QBF $\forall c \exists a \exists b((a \vee b) \leftrightarrow c)$ is valid and one of its possible model is $\psi_{\hat{a}}$; $\psi_{\hat{b}}$ with $\psi_{\hat{a}}=c$ and $\psi_{\hat{b}}=\perp$ (since $\left[a \leftarrow \psi_{\hat{a}}\right]\left[b \leftarrow \psi_{\hat{b}}\right]((b \vee a) \leftrightarrow c)=((\perp \vee c) \leftrightarrow c)$ is a tautology). A (Boolean) model of an unquantified Boolean formula corresponds exactly to a (QBF) model of its existential closure. A QBF is valid if and only if there exists a model. An interpretation $v$ is said to be in a model $\psi_{\hat{y_{1}}} ; \ldots ; \psi_{\hat{y_{p}}}$ if for every $i, 1 \leq i \leq n, v^{*}\left(\psi_{\hat{y}_{i}}\right)=v\left(y_{i}\right)$; for example the interpretation $v=$ $\{c, a, \neg b\}$ is in the above model since $v^{*}\left(\psi_{\hat{a}}\right)=$ true $=v(a)$ and $v^{*}\left(\psi_{\hat{b}}\right)=$ false $=v(b)$ but $v^{\prime}=\{\neg c, a, \neg b\}$ is not in it since $v^{\prime *}\left(\psi_{\hat{a}}\right)=$ false $\neq v^{\prime}(a)$. Modelequivalence for QBF is defined in [25] as follows : Two QBF $F$ and $F^{\prime}$ are modelequivalent (denoted $F \cong F^{\prime}$ ) if every model of $F$ is a model of $F^{\prime}$ and conversely; this equivalence is about preservation of models and not only preservation of validity; for example, $\forall c \exists a \exists b((a \vee b) \leftrightarrow c) \equiv \top$ but $\forall c \exists a \exists b((a \vee b) \leftrightarrow c) \not \top$.

\section{3 sat-certificate.}

A sat-certificate [3] for a QBF $F$, with $y_{1}, \ldots, y_{p}$ its existentially quantified variables, is a sequence of pairs of formulae $\left(\phi_{1}, \nu_{1}\right) ; \ldots ;\left(\phi_{p}, \nu_{p}\right), \phi_{i}$ and $\nu_{i}$ de- 
fined over the universally quantified variables of $F$ preceding the variable $y_{i}$, $1 \leq i \leq p$. It is defined in [3] only for CNF QBF with sequences of pairs of binary decision diagrams (BDD) [8]. A sat-certificate is consistent if for every $i, 1 \leq i \leq p,\left(\phi_{i} \wedge \nu_{i}\right) \equiv \perp$. If a sat-certificate is consistent then $\phi_{1} ; \ldots ; \phi_{p}$ and $\neg \nu_{1} ; \ldots ; \neg \nu_{p}$ are two models of the certified QBF (not necessarily the same). To certify the validity of a CNF QBF $Q M$ with a sat-certificate $\left(\phi_{1}, \nu_{1}\right) ; \ldots$; $\left(\phi_{p}, \nu_{p}\right)$ we have to check if $\left[\neg x_{1} \leftarrow \nu_{1}\right]\left[x_{1} \leftarrow \phi_{1}\right] \ldots\left[\neg x_{p} \leftarrow \nu_{p}\right]\left[x_{p} \leftarrow \phi_{p}\right](M)$ is a tautology. If the verification fails either the $\mathrm{QBF}$ is non valid or the satcertificate is not correct; conversely, if the verification succeeds then the QBF is valid and the sat-certificate encodes a model [3]. For example, from [3], the sequence of pairs of formulae $\left(\phi_{c}, \nu_{c}\right) ;\left(\phi_{e}, \nu_{e}\right) ;\left(\phi_{f}, \nu_{f}\right)$ with $\phi_{c}=\neg a, \nu_{c}=a$, $\phi_{e}=(a \wedge b \wedge d) \vee(\neg a \wedge \neg d), \nu_{e}=(\neg a \wedge d) \vee(a \wedge \neg d), \phi_{f}=(a \wedge b \wedge \neg d) \vee(\neg a \wedge b \wedge d)$ and $\nu_{f}=(\neg a \wedge \neg d)$ is a sat-certificate for the CNF QBF

$$
\xi=\forall a \forall b \exists c \forall d \exists e \exists f \mu
$$

with

$$
\begin{aligned}
\mu= & {[(\neg b \vee e \vee f) \wedge(a \vee c \vee f) \wedge(a \vee d \vee e) \wedge(\neg a \vee \neg b \vee \neg d \vee e) \wedge(\neg a \vee b \vee \neg c)} \\
& \wedge(\neg a \vee \neg c \vee \neg f) \wedge(a \vee \neg d \vee \neg e) \wedge(\neg a \vee d \vee \neg e) \wedge(a \vee \neg e \vee \neg f)] .
\end{aligned}
$$

This sat-certificate certifies the validity of this CNF QBF since $\left[\neg c \leftarrow \nu_{c}\right][c \leftarrow$ $\left.\phi_{c}\right]\left[\neg e \leftarrow \nu_{e}\right]\left[e \leftarrow \phi_{e}\right]\left[\neg f \leftarrow \nu_{f}\right]\left[f \leftarrow \phi_{f}\right](M)$ is a tautology. One can remark that this sat-certificate is consistent and that

$$
\phi_{c} ; \phi_{e} ; \phi_{f}=\neg a ;(a \wedge b \wedge d) \vee(\neg a \wedge \neg d) ;(a \wedge b \wedge \neg d) \vee(\neg a \wedge b \wedge d)
$$

and $\neg \nu_{c} ; \neg \nu_{e} ; \neg \nu_{f}=\neg a ; \neg(\neg a \wedge d) \vee(a \wedge \neg d) ; \neg(\neg a \wedge \neg d)$ are a two different models for the $\mathrm{QBF} \xi$.

\section{Literal Base}

In this section we present formally our proposal for a unified framework for certificate and compilation for QBF: the literal base representation. This representation extends the sat-certificate representation of [3].

Definition 1 (Literal base). A literal base is a pair $(Q, G)$ constituted

- either of $Q=\epsilon$ and $G=\top$ or $G=\perp$;

- either of a binder $Q=q_{1} x_{1} \ldots q_{n} x_{n}, n>0$, and a sequence of pairs of formulae $G=\left(P_{1}, N_{1}\right) ; \ldots ;\left(P_{n}, N_{n}\right)$ such that the formulae $P_{k}$ and $N_{k}$, called guards, are only built on the variables $\left\{x_{1}, \ldots, x_{k-1}\right\}$ (or $\top$ or $\perp$ when $k=1$ ).

We denote $\mathcal{B}_{Q}$ the set of the literal bases for a binder $Q, L B=\bigcup_{Q} \mathcal{B}_{Q}$ the literal base language and define the function grds such that $\operatorname{grds}((Q, G))=G$. 
A literal base is an explicit representation in the order of the binder of the dependencies that have to exist between an existentially quantified variable and the variables preceding it.

By the latter definition:

- if $Q=\epsilon$ then $\mathcal{B}_{\epsilon}=\{(\epsilon, \top),(\epsilon, \perp)\}$;

- if $Q=q x$ then $\mathcal{B}_{q x}=\{(q x,(\top, \top)),(q x,(\top, \perp)), \quad(q x,(\perp, \top)),(q x,(\perp, \perp))\}$

If $n$ is the number of variables of a binder $Q$ then the size of $\mathcal{B}_{Q}$ is $\underbrace{2^{2 \cdots}}_{n+1}$.

We interpret a literal base language as a representation for a subset of the QBF language.

Definition 2 (Interpretation of a literal base). The interpretation function is a function from $L B$ to $Q B F$ denoted $(.)^{*}$ and is defined as follows :

- if $l b=(\epsilon, G)$ then $l b^{*}=G$;

- if $l b=\left(q_{1} x_{1} \ldots q_{n} x_{n},\left(P_{1}, N_{1}\right) ; \ldots ;\left(P_{n}, N_{n}\right)\right), n>0$, then

$$
l b^{*}=q_{1} x_{1} \ldots q_{n} x_{n} \bigwedge_{k \leq n}\left(\left(\neg x_{k} \vee P_{k}\right) \wedge\left(x_{k} \vee N_{k}\right)\right)
$$

If $X$ is a subset of $B L$ then $X^{*}$ denotes $\left\{l b^{*} \mid l b \in X\right\}$. From here non_valid denotes a literal base whose interpretation is non valid and we extend the latter definition by non_valid ${ }^{*}=\perp$.

Clearly enough from Defintion 2 , if a literal base $\left(Q,\left(P_{1}, N_{1}\right) ; \ldots ;\left(P_{n}, N_{n}\right)\right)$ is such that its interpretation is valid then necessarily for every universally quantified variable $x_{i}, P_{i}$ and $N_{i}$ can be replaced in the literal base by $T$. The following literal base

$$
\beta=\left(\forall a \forall b \exists c \forall d \exists e \exists f,(\top, \top) ;(\top, \top) ;\left(P_{c}, N_{c}\right) ;(\top, \top) ;\left(P_{e}, N_{e}\right) ;\left(P_{f}, N_{f}\right)\right)
$$

with $P_{c}=\neg a, N_{c}=a, P_{e}=(\neg d \wedge c \wedge \neg a) \vee(d \wedge \neg c \wedge a)$,

$$
\begin{gathered}
N_{e}=(d \wedge c \wedge \neg a) \vee(\neg c \wedge \neg b \wedge a) \vee(\neg d \wedge \neg c \wedge a), \\
P_{f}=(\neg e \wedge d \wedge c \wedge \neg a) \vee(\neg e \wedge \neg c \wedge \neg b \wedge a) \vee \\
(d \wedge \neg c \wedge \neg b \wedge a) \vee(\neg e \wedge \neg d \wedge \neg c \wedge a) \vee(e \wedge d \wedge \neg c \wedge a)
\end{gathered}
$$

and

$$
\begin{aligned}
& N_{f}=(\neg e \wedge d \wedge c \wedge \neg b \wedge \neg a) \vee(e \wedge \neg d \wedge c \wedge \neg a) \vee \\
& (\neg e \wedge \neg c \wedge \neg b \wedge a) \vee(d \wedge \neg c \wedge \neg b \wedge a) \vee(e \wedge d \wedge \neg c \wedge a),
\end{aligned}
$$

is such that its interpretation is model-equivalent to (1) (i.e. $\beta^{*} \cong F$ ).

The following theorem establishes that for every QBF there exists a literal base such that its interpretation is model-equivalent to the QBF. By this theorem the literal base language may be considered as a target compilation language for QBF. 
Theorem 1 (Completeness of $L B$ ). Let $Q M$ be a $Q B F$. Then there exists a literal base $l b \in \mathcal{B}_{Q}$ such that $l b^{*} \cong Q M$.

A sat-certificate for a QBF is easily extended to a literal base: we add the binder of the QBF and in the sequence of the sat-certificate for each universally quantified variable we add a couple $(T, T)$. Hence, the interpretation of the satcertificate considered as a literal base has only one model which is the model of the QBF. In a sat-certificate $\left(\phi_{1}, \nu_{1}\right) ; \ldots ;\left(\phi_{p}, \nu_{p}\right)$ formulae $\phi_{i}$ and $\nu_{i}, 1 \leq i \leq p$, only depend on the preceding universally quantified variables while in a literal base $\left(Q,\left(P_{1}, N_{1}\right) ; \ldots ;\left(P_{n}, N_{n}\right)\right)$ formulae $P_{i}$ and $N_{i}, 1 \leq i \leq n$, may depend on all the preceding variables.

The propositional fragment in which the propositional formulae of the literal bases are defined needs only to be complete and may be chosen w.r.t. its succinctness (see [14] for a survey on properties of propositional fragments).

When a QBF is considered to model a finite two-player game, the validity of the QBF means that the "existential" player is sure to win if he follows the moves obtained from the (sequence of formulae of the) model. We are interested in the following question: since until now I have followed a (sequence of formulae of a) model, can I change my mind for the next move? We call this problem the "next move choice problem" and we define it formally.

Definition 3 (Next move choice problem for a subset $X$ of QBF).

- Instance : A formula $q_{1} x_{1} \ldots q_{n} x_{n} M$ from a subset $X$ of $Q B F$, a sequence of substitutions $\left[x_{1} \leftarrow C_{1}\right] \ldots\left[x_{i} \leftarrow C_{i}\right]$ obtained from a (sequence of formulae of a) model for $q_{1} x_{1} \ldots q_{n} x_{n} M$ with $q_{i}=\exists$ and $C_{1}, \ldots, C_{i} \in\{\top, \perp\}$.

- Query: Does there exist a model for

$q_{i+1} \ldots q_{n} x_{n}\left[x_{1} \leftarrow C_{1}\right] \ldots\left[x_{i-1} \leftarrow C_{i-1}\right]\left[x_{i} \leftarrow \overline{C_{i}}\right](M)$.

Clearly enough, the next move choice problem is still PSPACE-complete if we consider $X=Q B F$.

Considering again the QBF (1) and one of its model (2), we know that $\forall d \exists e \exists f[a \leftarrow \top][b \leftarrow \top][c \leftarrow \perp](\mu)$ is valid (since $[a \leftarrow \top][b \leftarrow \top]\left(\phi_{c}\right) \equiv \perp$ ) but is $\forall d \exists e \exists f[a \leftarrow \top][b \leftarrow \top][c \leftarrow \top](\mu)$ also valid?

We introduce a new property, called "optimality", for literal bases in order to exhibit a QBF fragment in which the next move choice problem is polytime w.r.t the size of the literal base.

Definition 4 (Optimality of a literal base). Let $l b$ be a literal base such that $l b=\left(q_{1} x_{1} \ldots q_{n} x_{n},\left(P_{1}, N_{1}\right) ; \ldots ;\left(P_{n}, N_{n}\right)\right)$ and $l b^{*}=q_{1} x_{1} \ldots q_{n} x_{n} G$. The literal base $l b$ is optimal if the following holds. For all $i, 1 \leq i \leq n$, let $\left[x_{1} \leftarrow\right.$ $\left.C_{1}\right] \ldots\left[x_{i-1} \leftarrow C_{i-1}\right]$ be an interpretation such that for all $k, 1 \leq k<i$ if $C_{k}=\top$ then $=\left[x_{1} \leftarrow C_{1}\right] \ldots\left[x_{k-1} \leftarrow C_{k-1}\right]\left(P_{k}\right)$ else $\models\left[x_{1} \leftarrow C_{1}\right] \ldots\left[x_{k-1} \leftarrow\right.$ $\left.C_{k-1}\right]\left(N_{k}\right)$.

Then

$$
\begin{aligned}
& =\left[x_{1} \leftarrow C_{1}\right] \ldots\left[x_{i-1} \leftarrow C_{i-1}\right]\left(P_{i}\right) \\
& \text { if and only if there exists a model for } \\
& q_{i+1} x_{i+1} \ldots q_{n} x_{n}\left[x_{1} \leftarrow C_{1}\right] \ldots\left[x_{i-1} \leftarrow C_{i-1}\right]\left[x_{i} \leftarrow \top\right](G)
\end{aligned}
$$


and

$$
\begin{aligned}
& \models\left[x_{1} \leftarrow C_{1}\right] \ldots\left[x_{i-1} \leftarrow C_{i-1}\right]\left(N_{i}\right) \\
& \text { if and only if there exists a model for } \\
& q_{i+1} x_{i+1} \ldots q_{n} x_{n}\left[x_{1} \leftarrow C_{1}\right] \ldots\left[x_{i-1} \leftarrow C_{i-1}\right]\left[x_{i} \leftarrow \perp\right](G) .
\end{aligned}
$$

We denote by $O B L$ the set of optimal literal bases.

Considering again (3), $\beta$ is an optimal literal base. Since the interpretation of $\beta$ is model-equivalent to (2) (i.e. $\forall a \forall b \exists c \forall d \exists e \exists f \mu$ ) and $[a \leftarrow \top][b \leftarrow \top]\left(N_{c}\right) \equiv \perp$ the QBF $\forall d \exists e \exists f[a \leftarrow \top][b \leftarrow \top][c \leftarrow \top](\mu)$ is not valid.

The most important property of optimal literal bases is that the next move choice problem is polytime and no more PSPACE-complete.

Theorem 2. The next move choice problem for $O B L^{*}$ is polytime.

If a QBF modeling a finite two-player game is compiled off-line in an optimal literal base, the computation of any sequence of moves leading to victory is polytime. An optimal literal base may be seen as a dynamic decision tree. The property of optimality of a literal base is linked with the property of minimality of a QBF which expresses that the QBF matrix contains only the models needed by the $(\mathrm{QBF})$ models.

Definition 5 (Minimality of a QBF). A QBF is minimal if all the (propositional) models of the matrix are (at least) in one of its (QBF) model.

For example, the QBF $\exists a \forall b((a \wedge b) \vee(a \wedge \neg b) \vee(\neg a \wedge b))$ is not minimal since the (Boolean) model $\{\neg a, b\}$ of the matrix is not in the only one model $\psi_{\hat{a}}=T$.

Theorem 3. Let lb be an optimal literal base. Then $l b^{*}$ is a minimal QBF.

The converse of Theorem 3 is false: The literal base $(\exists a \forall b,(\top, \top),(a, a))$ is not optimal (since there is no model with $\psi_{\hat{a}}=\perp$ ) but its interpretation is minimal.

\section{Literal base and sat-certificate for search-based algorithms}

In this section we are interested in the following problem: how to extend a search-based procedure in order to compute directly the sat-certificate and not a posteriori from a trace. To do this we define an operator for literal bases in order to be able to build a sat-certificate from the sat-certificates of its two simpler subproblems.

Definition 6. The operator $\circ_{x}: \mathcal{B}_{Q} \times \mathcal{B}_{Q} \rightarrow \mathcal{B}_{\forall x Q}$ is defined as follows :

$$
\begin{aligned}
& \left(Q,\left(P_{1}, N_{1}\right) ; \ldots ;\left(P_{n}, N_{n}\right)\right) \circ_{x}\left(Q,\left(P_{1}^{\prime}, N_{1}^{\prime}\right) ; \ldots ;\left(P_{n}^{\prime}, N_{n}^{\prime}\right)\right) \\
& =(\forall x Q,(\top, \top) ; \\
& \quad\left(\left(\left(\neg x \vee P_{1}\right) \wedge\left(x \vee P_{1}^{\prime}\right)\right),\left(\left(\neg x \vee N_{1}\right) \wedge\left(x \vee N_{1}^{\prime}\right)\right)\right) ; \ldots ; \\
& \left.\quad\left(\left(\left(\neg x \vee P_{n}\right) \wedge\left(x \vee P_{n}^{\prime}\right)\right),\left(\left(\neg x \vee N_{n}\right) \wedge\left(x \vee N_{n}^{\prime}\right)\right)\right)\right)
\end{aligned}
$$


In this definition, if $x$ is interpreted to true (resp. false) then for all $i, 1 \leq i \leq$ $n,\left(\left(\neg x \vee P_{i}\right) \wedge\left(x \vee P_{i}^{\prime}\right)\right) \equiv P_{i}\left(\right.$ resp. $\left.P_{i}^{\prime}\right)$ and $\left(\left(\neg x \vee N_{i}\right) \wedge\left(x \vee N_{i}^{\prime}\right)\right) \equiv N_{i}$ (resp. $\left.N_{i}^{\prime}\right)$. If $\left(Q,\left(P_{1}, N_{1}\right) ; \ldots ;\left(P_{n}, N_{n}\right)\right)$ and $\left(Q,\left(P_{1}^{\prime}, N_{1}^{\prime}\right) ; \ldots ;\left(P_{n}^{\prime}, N_{n}^{\prime}\right)\right)$ are sat-certificates and $Q=q_{1} x_{1} \ldots q_{n} x_{n}$ with $q_{i}=\forall$ then clearly enough $\left(\left(\neg x \vee P_{i}\right) \wedge\left(x \vee P_{i}^{\prime}\right)\right) \equiv$ $\top \equiv\left(\left(\neg x \vee N_{i}\right) \wedge\left(x \vee N_{i}^{\prime}\right)\right)$.

We establish by the following theorem that the $\circ$ operator composes two sat-certificates in a new sat-certificate.

Theorem 4. Let $\forall x Q M$ be a $Q B F$. If $l b_{\top}$ is a sat-certificate for $Q[x \leftarrow \top](M)$ and $l b_{\perp}$ is a sat-certificate for $Q[x \leftarrow \perp](M)$ then $\left(l b_{\top} \circ_{x} l b_{\perp}\right)$ is a sat-certificate for $\forall x Q M$.

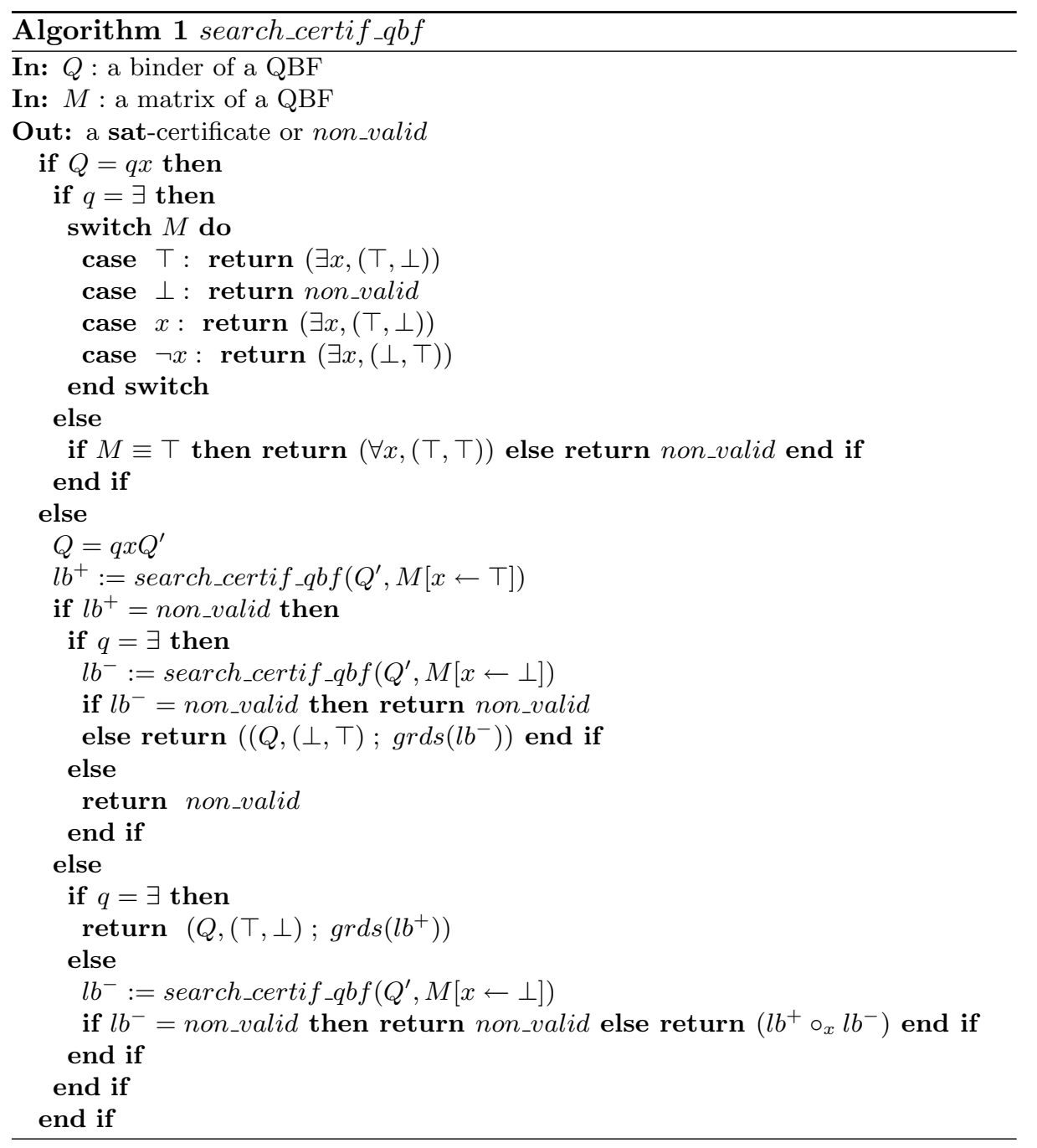


We are now able to present the search-based algorithm search_certif_qbf which computes a sat-certificate for a QBF. The search_certif_qbf algorithm checks first if the binder is reduced to a single quantifier with its variable. In this case, if it is an existential quantifier four cases are possible, corresponding, in the order of the algorithm, to : $\exists x \top \equiv \exists x x^{1}, \exists x \perp \equiv \perp, \exists x x \cong \exists x((\neg x \vee \top) \wedge(x \vee \perp))$ and $\exists x \neg x \cong \exists x((\neg x \vee \perp) \wedge(x \vee \top))$. If the quantifier is universal then if $M \equiv \top$ then $\forall x M \equiv \top$ else $\forall x x \equiv \forall x \neg x \equiv \forall x \perp \equiv \perp$. If there are some quantifiers, since the algorithm is a search-based one, the most external quantifier is considered. If this quantifier is existential then if one of the recursive calls for the substitution by $\top$ (resp. $\perp$ ) for the variable $x$ is different to non_valid the returned satcertificate is $\left(Q,(\top, \perp) ; \operatorname{grds}\left(l b^{+}\right)\right)$(resp. $\left.\left(Q,(\perp, \top) ; \operatorname{grds}\left(l b^{-}\right)\right)\right)$which expresses that $x$ must be true (resp. false). If the quantifier is universal then if at least one recursive call for the substitution by $\top$ or by $\perp$ for the variable $x$ returns non_valid then non_valid is returned otherwise the Skolem functions of the two sat-certificates have to be combined to integrate the new argument $x$ by $\left(l b^{+} \circ_{x}\right.$ $l b^{-}$) before this new sat-certificate is returned.

Theorem 5 (Correctness of search_certif_qbf). Let $Q M$ be a $Q B F$. search_certif_qbf $(Q, M)$ returns a sat-certificate for $Q M$ if the $Q B F$ is valid and non_valid otherwise.

In case of search-based algorithms for CNF QBF, unit propagation and monotone literal propagation [11] may be easily added the search_certif_qbf algorithm.

\section{$5 \quad$ Literal bases and QBF compilation for search-based algorithms}

Since Theorem 1 establishes the completeness of the literal base language, $L B$ may be considered as a target language for the compilation of a QBF. In this section we are interested in the following problem: how to extend a search-based procedure in order to compile a QBF in an optimal literal base. To do this we define an operator for literal bases which compile a QBF by the combination of the results of the compilation of its two simpler subproblems.

Definition 7. Let $Q^{\prime}=q_{2} x_{2} \ldots q_{n} x_{n}$ and $Q=q_{1} x_{1} Q^{\prime}$ be two binders and $l b, l b^{\prime} \in \mathcal{B}_{Q}$. The operator $\oplus: \mathcal{B}_{Q} \times \mathcal{B}_{Q} \rightarrow \mathcal{B}_{Q}$ is defined as follows :

If $Q=\epsilon$ then $\left(l b \oplus l b^{\prime}\right)=\left(l b^{*} \vee l b^{* *}\right)$ else

$$
\begin{aligned}
(Q, & \left.\left(P_{1}, N_{1}\right) ; \ldots ;\left(P_{n}, N_{n}\right)\right) \oplus\left(Q,\left(P_{1}^{\prime}, N_{1}^{\prime}\right) ; \ldots ;\left(P_{n}^{\prime}, N_{n}^{\prime}\right)\right) \\
=(Q, & \left(\left(P_{1} \vee P_{1}^{\prime}\right),\left(N_{1} \vee N_{1}^{\prime}\right)\right) ; \\
& \left(\mathcal{P}_{2} \wedge\left(P_{2}^{\prime} \vee \mathcal{X}\right) \wedge\left(P_{2} \vee \mathcal{X}^{\prime}\right), \mathcal{N}_{2} \wedge\left(N_{2}^{\prime} \vee \mathcal{X}\right) \wedge\left(N_{2} \vee \mathcal{X}^{\prime}\right)\right) ; \ldots ; \\
& \left.\left(\mathcal{P}_{n} \wedge\left(P_{n}^{\prime} \vee \mathcal{X}\right) \wedge\left(P_{n} \vee \mathcal{X}^{\prime}\right), \mathcal{N}_{n} \wedge\left(N_{n}^{\prime} \vee \mathcal{X}\right) \wedge\left(N_{n} \vee \mathcal{X}^{\prime}\right)\right)\right)
\end{aligned}
$$

\footnotetext{
${ }^{1}$ Since we need one solution, we privilege the interpretation of $x$ to true
} 
with $\mathcal{X}=\left(\left(\neg x_{1} \vee P_{1}\right) \wedge\left(x_{1} \vee N_{1}\right)\right), \mathcal{X}^{\prime}=\left(\left(\neg x_{1} \vee P_{1}^{\prime}\right) \wedge\left(x_{1} \vee N_{1}^{\prime}\right)\right)$ and the recursive call:

$$
\begin{aligned}
& \left(Q^{\prime},\left(\mathcal{P}_{2}, \mathcal{N}_{2}\right) ; \ldots ;\left(\mathcal{P}_{n}, \mathcal{N}_{n}\right)\right)= \\
& \quad\left(Q^{\prime},\left(P_{2}, N_{2}\right) ; \ldots ;\left(P_{n}, N_{n}\right)\right) \oplus\left(Q^{\prime},\left(P_{2}^{\prime}, N_{2}^{\prime}\right) ; \ldots ;\left(P_{n}^{\prime}, N_{n}^{\prime}\right)\right)
\end{aligned}
$$

This operator is the counterpart of the disjunction for the QBF. In the previous definition when $n=1$,

$$
\left(q_{1} x_{1},\left(P_{1}, N_{1}\right)\right) \oplus\left(q_{1} x_{1},\left(P_{1}^{\prime}, N_{1}^{\prime}\right)\right)=\left(q_{1} x_{1},\left(\left(P_{1} \vee P_{1}^{\prime}\right),\left(N_{1} \vee N_{1}^{\prime}\right)\right)\right)
$$

which defines the base case of recursivity of $\oplus$. We develop for the case $n=2$ the disjunction of the matrices of the interpretation of two literal bases and show how we can find back Definition 7: Since $\left(q_{2} x_{2},\left(P_{2}, N_{2}\right)\right) \oplus\left(q_{2} x_{2},\left(P_{2}^{\prime}, N_{2}^{\prime}\right)\right)=$ $\left(q_{2} x_{2},\left(\left(P_{2} \vee P_{2}^{\prime}\right),\left(N_{2} \vee N_{2}^{\prime}\right)\right)\right), \mathcal{P}_{2}=\left(P_{2} \vee P_{2}^{\prime}\right)$ and $\mathcal{N}_{2}=\left(N_{2} \vee N_{2}^{\prime}\right)$ then

$$
\begin{aligned}
( & \left.\left(\neg x_{1} \vee P_{1}\right) \wedge\left(x_{1} \vee N_{1}\right)\right) \wedge\left(\left(\neg x_{2} \vee P_{2}\right) \wedge\left(x_{2} \vee N_{2}\right)\right) \vee \\
( & \left.\left(\neg x_{1} \vee P_{1}^{\prime}\right) \wedge\left(x_{1} \vee N_{1}^{\prime}\right)\right) \wedge\left(\left(\neg x_{2} \vee P_{2}^{\prime}\right) \wedge\left(x_{2} \vee N_{2}^{\prime}\right)\right) \\
\equiv & \left(\neg x_{1} \vee\left(P_{1} \vee P_{1}^{\prime}\right)\right) \wedge\left(x_{1} \vee\left(N_{1} \vee N_{1}^{\prime}\right)\right) \wedge \\
& \left(\neg x_{2} \vee\left(\left(P_{2} \vee P_{2}^{\prime}\right) \wedge\left(P_{2}^{\prime} \vee\left(\left(\neg x_{1} \vee P_{1}\right) \wedge\left(x_{1} \vee N_{1}\right)\right)\right) \wedge\left(P_{2} \vee\left(\left(\neg x_{1} \vee P_{1}^{\prime}\right) \wedge\left(x_{1} \vee N_{1}^{\prime}\right)\right)\right)\right)\right) \wedge \\
& \left(\neg x_{2} \vee\left(\left(N_{2} \vee N_{2}^{\prime}\right) \wedge\left(N_{2}^{\prime} \vee\left(\left(\neg x_{1} \vee P_{1}\right) \wedge\left(x_{1} \vee N_{1}\right)\right)\right) \wedge\left(N_{2} \vee\left(\left(\neg x_{1} \vee P_{1}^{\prime}\right) \wedge\left(x_{1} \vee N_{1}^{\prime}\right)\right)\right)\right)\right) \\
\equiv & \left(\neg x_{1} \vee\left(P_{1} \vee P_{1}^{\prime}\right)\right) \wedge\left(x_{1} \vee\left(N_{1} \vee N_{1}^{\prime}\right)\right) \wedge \\
& \left(\neg x_{2} \vee\left(\mathcal{P}_{2} \wedge\left(P_{2}^{\prime} \vee \mathcal{X}\right) \wedge\left(P_{2} \vee \mathcal{X}^{\prime}\right)\right)\right) \wedge\left(\neg x_{2} \vee\left(\mathcal{N}_{2} \wedge\left(N_{2}^{\prime} \vee \mathcal{X}\right) \wedge\left(N_{2} \vee \mathcal{X}^{\prime}\right)\right)\right)
\end{aligned}
$$

Definition 7 may be improved with no cost by applying as simplification rules some usual logical equivalences: $(x \wedge x) \equiv x,(x \vee x) \equiv x,(x \wedge \neg x) \equiv \perp$ and $(x \vee \neg x) \equiv \top$ with $x$ a propositional variable; $(H \wedge \top) \equiv H,(H \wedge \perp) \equiv \perp$, $(H \vee \top) \equiv \top$ and $(H \vee \perp) \equiv H$ with $H$ a propositional formula.

Theorem 6. Let $Q$ be a binder and $l b, l b^{\prime} \in \mathcal{B}_{Q}$ such that $l b^{*}=Q M$ and $l b^{\prime *}=Q M^{\prime}$. Then $\left(l b \oplus l b^{\prime}\right)^{*}=Q M_{\oplus}$ with $M_{\oplus} \equiv\left(M \vee M^{\prime}\right)$.

We are now able to present the search-based algorithm search_comp_qbf which compiles a QBF into an optimal literal base. The search_comp_qbf algorithm checks first if the binder is reduced to a single quantifier with its variable. If it is the case and if $M \equiv T$, conversely to search_certif_qbf algorithm, $(T, T)$ is returned (since $\exists x \top \cong \exists x((\neg x \vee \top) \wedge(x \vee \top)))$ in order to compose the two possibilities. If there are some quantifiers, since the algorithm is a search-based one, the first one is considered. Following semantics of QBF, if there is no model for one (resp. both) recursive call then there is no model for the QBF if the quantifier is universal (resp. existential) ; if there are models for both recursive calls then, for both quantifiers, $\left(Q,(\top, \perp) ; \operatorname{grds}\left(l b^{+}\right)\right) \oplus\left(Q,(\perp, \top) ; \operatorname{grds}\left(l b^{-}\right)\right.$ is returned.

Literal bases generated by the search_comp_qbf compilation algorithm may be in worst case of exponential size.

Theorem 7 (Correctness of search_comp_qbf). Let $Q M$ be a $Q B F$. search_comp_qbf $(Q, M)$ returns a literal base lb such that $l b^{*} \cong Q M$ if $Q M$ is valid and returns non_valid otherwise. 


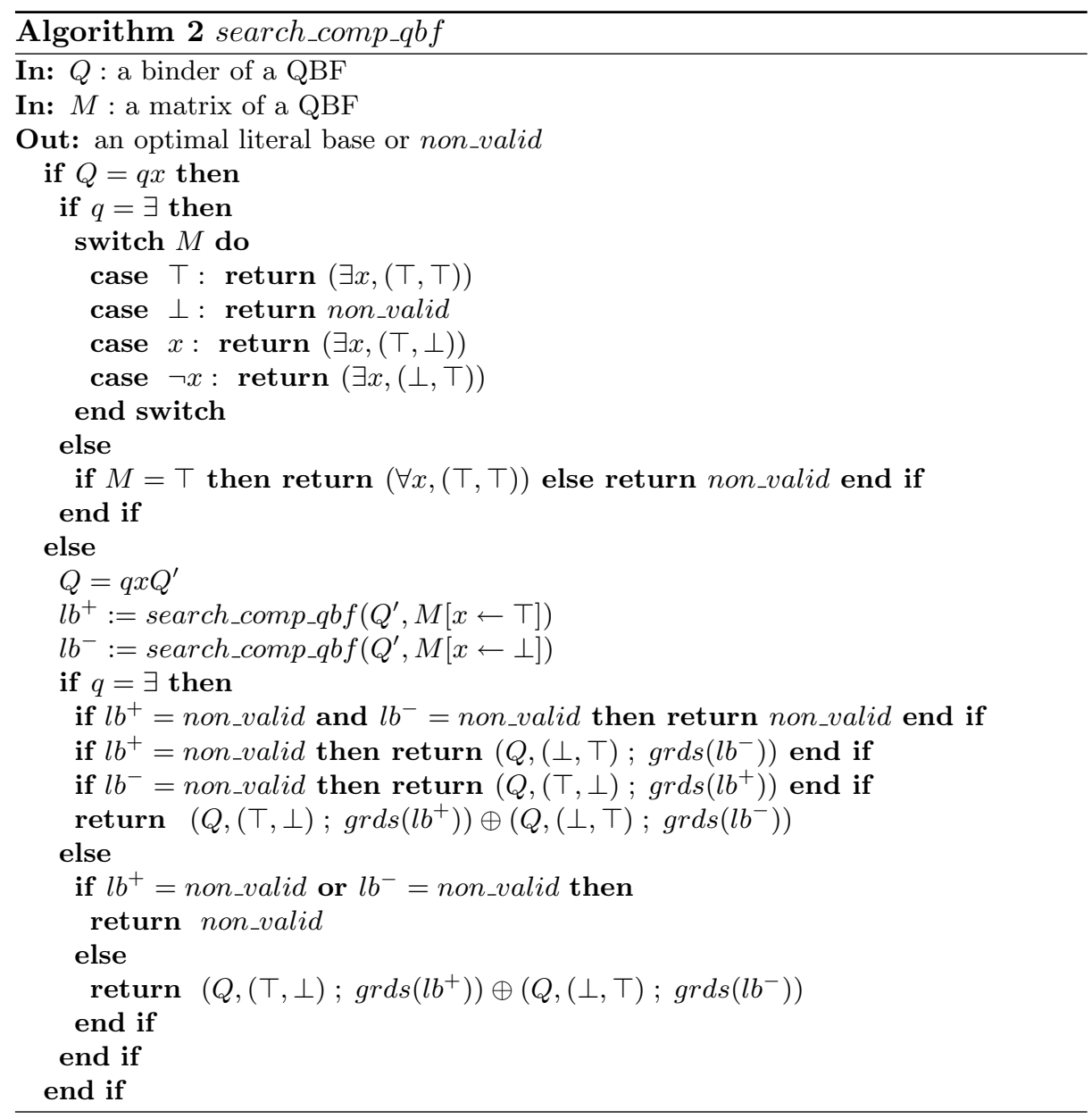

We can now establish that the literal base generated by the search_comp_qbf algorithm is optimal.

Theorem 8 (Optimality of search_comp_qbf). Let $Q M$ be a valid $Q B F$. Then search_comp_qbf $(Q, M)$ is optimal.

In case of search-based algorithms for CNF QBF, unit propagation may be easily added ; but, conversely to search_certif_qbf algorithm, monotone literal propagation can not be applied to the search_comp_qbf algorithm since it does not preserve all the models. 


\section{Related work}

QBF certificates. To the best of our knowledge, there exist only two suggestions for QBF certificates and methods to generate them ${ }^{2}$. The first approach [20] is a method to generate a list of pairs of the form $\left(v, f_{v}\right)$ where $f_{v}$ are the Skolem functions for fresh variables $v$ from the classical extension rule for propositional logic [26]. The second approach proposed in $[4,3]$ introduces the sat-certificate. It is described independently of any algorithm, but with binary decision diagrams (BDD) [8] and only for formulae in CNF. The computation of a sat-certificate is described in [2] in the framework of sKizzo as a reconstruction from a trace: the "inference log". An external certifier application (ozziKs) is charged with interpreting the content of the log in order to construct certificates [4]. Since the solver can choose between five different inference strategies there are many different kinds of instructions in the inference logs. It results in the need for a heavyweight proof checker. This approach is based on a trace of what the solver is doing and it probably does not scale well because of the growth of this trace. It can take more time to generate the sat-certificate from the trace than it took to generate the model [4].

QBF compilation. Knowledge compilation with a subset of the propositional language as a target language has been widely study (see [14] for a "knowledge compilation map"), but it is not the case for QBF compilation: [16] focuses on selected propositional fragments and quantifier elimination while [12] focuses on complexity of QBF built on the same selected propositional fragments. The compiler for CNF QBF proposed in [25] extends a quantifier-elimination decision procedure [22] as follows: for a CNF QBF $q_{1} x_{1} \ldots q_{n-1} x_{n-1} q_{n} x_{n} M$, we compute the formulae $M_{n}, P_{n}$ and $N_{n}$ defined on $\left\{x_{1}, \ldots, x_{n-1}\right\}$ such that $M \equiv$ $\left(M_{n} \wedge\left(\left(\neg x_{n} \vee P_{n}\right) \wedge\left(x_{n} \vee N_{n}\right)\right)\right)$; if $q_{n}=\exists$ then the process is recursively called on $q_{1} x_{1} \ldots q_{n-1} x_{n-1}\left(M_{n} \wedge\left(P_{n} \vee N_{n}\right)\right)$ otherwise the process is recursively called on $q_{1} x_{1} \ldots q_{n-1} x_{n-1}\left(M_{n} \wedge\left(P_{n} \wedge N_{n}\right)\right)$. The target language of this approach is similar to the literal base language: $\left(q_{1} x_{1} \ldots q_{n} x_{n},\left(P_{1}, N_{1}\right) ; \ldots ;\left(P_{n}, N_{n}\right)\right)$ is a literal base. Since $\left(P_{n} \vee N_{n}\right)$ is not CNF, the expansion of the existential quantifier for $\mathrm{CNF}$ is involved with a quadratic size increase of the formula [21]. Clearly enough the literal base generated by this quantifier-elimination compiler is optimal and it is usually smaller than the literal base generated by the search_comp_qbf with out simplifications.

\section{Concluding remarks}

We have described in this article a unified framework for sat-certificate and compilation of QBF. We have proposed a search-based procedure to compute

\footnotetext{
${ }^{2}$ The approach proposed in [27] is a method to generate a subset of the clauses of a QBF formula in prenex normal form which is non-valid from traces of search-based solvers. Since this approach is focused on non-validity, it is out of the scope of this paper which is focused on validity.
} 
sat-certificates which is very useful since most QBF solvers are search-based decision procedures.

Literal bases generated by the search_comp_qbf compilation algorithm may be in worst case of exponential size what complies with complexity results [13]. Anyway, we think that compilation is useful since all the solutions are kept and decision over existentially quantified variables may be not fully described in the QBF. In that case, for each existentially quantified variable, the two different possibilities are computed in polynomial time thanks to optimality and if both substitutions take part of a solution, the choice is left to the user, following its preferences.

Acknowledgement. We would like to thank the referees for their comments which helped improve this paper.

\section{References}

1. A. Ayari and D. Basin. Qubos: Deciding quantified boolean logic using propositional satisfiability solvers. In Proceedings of the 4 th International Conference on Formal Methods in Computer-Aided Design (FMCAD'02), pages 187-201, 2002.

2. M. Benedetti. Evaluating QBFs via Symbolic Skolemization. In Proceedings of the 11th International Conference on Logic for Programming, Artificial Intelligence, and Reasoning (LPAR'05), number 3452 in LNCS, pages 285-300. Springer, 2005.

3. M. Benedetti. Extracting certificates from quantified boolean formulas. In Proceedings of 9th International Joint Conference on Artificial Intelligence (IJCAI'05), pages 47-53, 2005.

4. M. Benedetti. skizzo: a suite to evaluate and certify QBFs. In Proceedings of the 20th International Conference on Automated Deduction (CADE'05), pages 369376, 2005.

5. M. Benedetti and H. Mangassarian. Experience and perspectives in qbf-based formal verification. Journal on Satisfiability, Boolean Modeling and Computation, 2008 (to appear).

6. A. Biere. Resolve and Expand. In Proceedings of the 7th International Confrerence on Theory and Applications of Satisfiability Testing (SAT'04), pages 59-70, 2004.

7. L. Bordeaux. Boolean and interval propagation for quantified constraints. In First International Workshop on Quantification in Constraint Programming, 2005.

8. R. E. Bryant. Graph-based algorithms for boolean function manipulation. IEEE Transactions on Computers, 35(8):677-691, 1986.

9. H. K. Büning, M. Karpinski, and A. Flögel. Resolution for quantified boolean formulas. Information and Computation, 117(1):12-18, 1995.

10. H. K. Büning, K. Subramani, and X. Zhao. Boolean functions as models for quantified boolean formulas. Journal of Automated Reasoning, 39(1):49-75, 2007.

11. M. Cadoli, M. Schaerf, A. Giovanardi, and M. Giovanardi. An algorithm to evaluate quantified boolean formulae and its experimental evaluation. Journal of Automated Reasoning, 28(2):101-142, 2002.

12. S. Coste-Marquis, D. Le Berre, F. Letombe, and P. Marquis. Propositional fragments for knowledge compilation and quantified boolean formulae. In Proceedings of the 20th National Conference on Artificial Intelligence (AAAI'05), pages 288$293,2005$. 
13. S. Coste-Marquis, H. Fargier, J. Lang, D. Le Berre, and P. Marquis. Representing policies for quantified boolean formulae. In Proceedings of the 10th International Conference on Principles of Knowledge Representation and Reasoning (KR'06), pages 286-296, 2006.

14. A. Darwiche and P. Marquis. A knowledge compilation map. Journal of Artificial Intelligence Research, 17:229-264, 2002.

15. M. Davis, G. Logemann, and D. Loveland. A machine program for theoremproving. Communication of the ACM, 5, 1962.

16. H. Fargier and P. Marquis. On the use of partially ordered decision graphs in knowledge compilation and quantified boolean formulae. In $A A A I, 2006$.

17. M. R. Garey and D. S. Johnson. Computers and Intractability; A Guide to the Theory of NP-Completeness. W. H. Freeman \& Co., 1990.

18. E. Giunchiglia, M. Narizzano, and A. Tacchella. Clause/term resolution and learning in the evaluation of quantified boolean formulas. Journal of Artificial Intelligence Research, 26:371-416, 2006.

19. F. Bacchus H. Samulowitz. Using SAT in QBF. In Proceedings of the 11th International Conference on Principles and Practice of Constraint Programming, Lecture Notes in Computer Science, volume 3709, pages 578 - 592, 2005.

20. T. Jussila, A. Biere, C. Sinz, D. Kröning, and C. Wintersteiger. A first step towards a unified proof checker for qbf. In Proceedings of the Tenth International Confrerence on Theory and Applications of Satisfiability Testing (SAT'07), pages 201-214, 2007.

21. F. Lonsing and A. Biere. Nenofex: Expanding NNF for QBF Solving. In Proceedings of the Eleventh International Conference on Theory and Applications of Satisfiability Testing (SAT'08), pages 196-210, 2008.

22. G. Pan and M.Y. Vardi. Symbolic Decision Procedures for QBF. In International Conference on Principles and Practice of Constraint Programming, 2004.

23. D.A. Plaisted, A. Biere, and Y. Zhu. A satisfiability procedure for quantified Boolean formulae. Discrete Applied Mathematics, 130:291-328, 2003.

24. J. Rintanen. Constructing conditional plans by a theorem-prover. Journal of Artificial Intelligence Research, 10:323-352, 1999.

25. I. Stéphan. Finding models for quantified boolean formulae. In First International Workshop on Quantification in Constraint Programming, 2005.

26. G. S. Tseitin. On the complexity of derivation in propositional calculus. In A. O. Slisenko, editor, Studies in Constructive Mathematics and Mathematical Logic, Part 2, pages 115-125. Consultants Bureau, New York, 1970.

27. Y. Yu and S. Malik. Validating the result of a quantified boolean formula (QBF) solver: theory and practice. In Proceedings of the 2005 conference on Asia South Pacific design automation (ASP-DAC '05), pages 1047-1051, 2005.

28. L. Zhang. Solving QBF with combined conjunctive and disjunctive normal form. In National Conference on Artificial Intelligence (AAAI'06), 2006. 


\section{Proofs}

By abuse of notation we define the interpretation function for guards as follows:

$$
\left(\left(P_{1}, N_{1}\right) ; \ldots ;\left(P_{n}, N_{n}\right)\right)^{*}=\bigwedge_{1 \leq k \leq n}\left(\left(\neg x_{k} \vee P_{k}\right) \wedge\left(x_{k} \vee N_{k}\right)\right)
$$

and the $\oplus$ operator only for guards as follows:

$$
\begin{aligned}
( & \left.\left(P_{1}, N_{1}\right) ; \ldots ;\left(P_{n}, N_{n}\right)\right) \oplus\left(\left(P_{1}^{\prime}, N_{1}^{\prime}\right) ; \ldots ;\left(P_{n}^{\prime}, N_{n}^{\prime}\right)\right) \\
= & \left(\left(P_{1} \vee P_{1}^{\prime}\right),\left(N_{1} \vee N_{1}^{\prime}\right)\right) ; \\
& \left(\mathcal{P}_{2} \wedge\left(P_{2}^{\prime} \vee \mathcal{X}\right) \wedge\left(P_{2} \vee \mathcal{X}^{\prime}\right), \mathcal{N}_{2} \wedge\left(N_{2}^{\prime} \vee \mathcal{X}\right) \wedge\left(N_{2} \vee \mathcal{X}^{\prime}\right)\right) ; \ldots ; \\
& \left.\left(\mathcal{P}_{n} \wedge\left(P_{n}^{\prime} \vee \mathcal{X}\right) \wedge\left(P_{n} \vee \mathcal{X}^{\prime}\right), \mathcal{N}_{n} \wedge\left(N_{n}^{\prime} \vee \mathcal{X}\right) \wedge\left(N_{n} \vee \mathcal{X}^{\prime}\right)\right)\right)
\end{aligned}
$$

with $\mathcal{X}=\left(\left(\neg x_{1} \vee P_{1}\right) \wedge\left(x_{1} \vee N_{1}\right)\right), \mathcal{X}^{\prime}=\left(\left(\neg x_{1} \vee P_{1}^{\prime}\right) \wedge\left(x_{1} \vee N_{1}^{\prime}\right)\right)$ and

$$
\left(\left(\mathcal{P}_{2}, \mathcal{N}_{2}\right) ; \ldots ;\left(\mathcal{P}_{n}, \mathcal{N}_{n}\right)\right)=\left(\left(P_{2}, N_{2}\right) ; \ldots ;\left(P_{n}, N_{n}\right)\right) \oplus\left(\left(P_{2}^{\prime}, N_{2}^{\prime}\right) ; \ldots ;\left(P_{n}^{\prime}, N_{n}^{\prime}\right)\right)
$$

Theorem 1 Let $Q M$ be a $Q B F$. Then there exists a literal base $l b \in \mathcal{B}_{Q}$ such that $l b^{*} \cong Q M$.

Proof of Theorem 1. This theorem is direct since every formula is equivalent to a conjunction of disjunctions of literals whose last variable can be distributed.

Theorem 2 The next move choice problem for $O B L^{*}$ is polytime.

Proof of Theorem 2. This theorem is a direct consequence of the definition of optimality for literal bases.

Theorem 3 Let $l b$ be an optimal literal base. Then $l b^{*}$ is a minimal QBF.

Proof of Theorem 3. If the interpretation of the literal base is not minimal then there exists a (propositional) model for the matrix which is not in a sequence of satisfying Skolem functions then there exists a sequence of guards satisfied by this model but at least one of this guard should not be satisfied since the literal base is not optimal.

Theorem 4 Let $\forall x Q M$ be a $Q B F$. If $l b_{\top}$ is a sat-certificate for $Q[x \leftarrow \top](M)$ and $l b_{\perp}$ is a sat-certificate for $Q[x \leftarrow \perp](M)$ then $\left(l b_{\top} \circ_{x} l b_{\perp}\right)$ is a sat-certificate for $\forall x Q M$.

Proof of Theorem 4. Let $\forall x_{1} q_{2} x_{2} \ldots q_{n} x_{n} M$ be a QBF.

Let $B_{\top}=\left(Q,\left(P_{2}, N_{2}\right) ; \ldots ;\left(P_{n}, N_{n}\right)\right)$ be a sat-certificate for $Q[x \leftarrow \top](M)$ and $B_{\perp}=\left(Q,\left(P_{2}^{\prime}, N_{2}^{\prime}\right) ; \ldots ;\left(P_{n}^{\prime}, N_{n}^{\prime}\right)\right)$ be a sat-certificate for $Q[x \leftarrow \perp](M)$. If $x_{i}$, $2 \leq i \leq n$, is a universally quantified variable then $P_{i}=N_{i}=P_{i}^{\prime}=N_{i}^{\prime}=\top$ then $\left(\left(\neg x_{i} \vee P_{i}\right) \wedge\left(x_{i} \vee P_{i}^{\prime}\right)\right) \equiv \top$ and $\left(\left(\neg x_{i} \vee N_{i}\right) \wedge\left(x_{i} \vee N_{i}^{\prime}\right)\right) \equiv \top$. If $x_{i}, 2 \leq i \leq n$, is 
an existentially quantified variable with its associated function $\hat{x}_{i}^{\top}$ for $B_{\top}$ (the sequence of these Boolean functions $\hat{x}_{i}^{\top}$ satisfies $\left.Q[x \leftarrow \top](M)\right)$ and with its associated function $\hat{x}_{i}^{\perp}$ for $B_{\perp}$ (the sequence of these Boolean functions $\hat{x}_{i}^{\perp}$ satisfies $Q[x \leftarrow \perp](M))$ then the interpretation $v$ associated to the Boolean function $\hat{x}_{i}$ such that $\hat{x}_{i}($ true $)=\hat{x}_{i}^{\top}$ and $\hat{x}_{i}($ false $)=\hat{x}_{i}^{\perp}$ verifies $v^{*}\left(\left(\left(\neg x_{i} \vee P_{i}\right) \wedge\left(x_{i} \vee P_{i}^{\prime}\right)\right)\right)=$ true and $v^{*}\left(\left(\left(\neg x_{i} \vee N_{i}\right) \wedge\left(x_{i} \vee N_{i}^{\prime}\right)\right)\right)=$ false. Moreover, the sequence of these Boolean functions $\hat{x}_{i}$ satisfies $\forall x_{1} q_{2} x_{2} \ldots q_{n} x_{n} M$.

Theorem 5 Let $Q M$ be a $Q B F$.

search_certif_qbf $(Q, M)$ returns a sat-certificate for $Q M$ if the $Q B F$ is valid and non_valid otherwise.

Proof of Theorem 5.

- Base case: Let suppose that $Q=\exists x$.

- If $M \equiv \top$ then search_certif_qbf $(\exists x, M)$ returns $B=(\exists x,(\top, \perp))$ then $B^{*}=\exists x((\neg x \vee \top) \wedge(x \vee \perp)) \cong \exists x x \equiv \exists x M$.

- If $M \equiv \perp$ then $\exists x M$ is not valid and search_certif_qbf $(\exists x, M)$ returns non_valid.

- If $M \equiv x$ then search_certif_qbf $(\exists x, M)$ returns $B=(\exists x,(\top, \perp))$ then $B^{*}=\exists x((\neg x \vee \top) \wedge(x \vee \perp)) \cong \exists x x \equiv \exists x M$.

- If $M \equiv \neg x$ then search_certif_qbf $(\exists x, M)$ returns $B=(\exists x,(\perp, \top))$ then $B^{*}=\exists x((\neg x \vee \perp) \wedge(x \vee \top)) \cong \exists x \neg x \equiv \exists x M$.

Now Let suppose that $Q=\forall x$. If $M \equiv \top$ then search_certif_qbf $(\forall x, M)$ returns $B=(\forall x,(\top, \top))$ then $B^{*}=\forall x((\neg x \vee \top) \wedge(x \vee \top)) \cong \forall x \top \cong \forall x M$. Else $\forall x M$ is not valid and search_certif_qbf( $\forall x, M)$ returns non_valid.

- Induction case: Let suppose that $Q=\exists x Q^{\prime}$.

Let

$$
b l^{+}=\text {search_certif_qbf }\left(Q^{\prime},[x \leftarrow \top](M)\right)
$$

and

$$
b l^{-}=\text {search_certif_qbf }\left(Q^{\prime},[x \leftarrow \perp](M)\right) .
$$

- We suppose that $b l^{+}=$non_valid and $b l^{-}=$non_valid then search_certif_qbf $\left(\exists x Q^{\prime}, M\right)$ returns non_valid. By induction hypothesis both QBF $Q^{\prime}[x \leftarrow \top](M)$ and $Q^{\prime}[x \leftarrow \perp](M)$ are not valid then by definition $\exists x Q^{\prime} M$ is not valid.

- We suppose that $b l^{+} \neq$non_valid (the case is similar for $\left(b l^{+}=\right.$non_valid

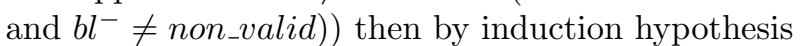

$b l^{+}=$search_certif_qbf $\left(Q^{\prime},[x \leftarrow \top](M)\right)$ is a sat-certificate for $Q^{\prime}[x \leftarrow$ $\top](M)$ and $\left(\exists x Q^{\prime},(\top, \perp), \operatorname{grds}\left(b l^{+}\right)\right)$is a sat-certificate for $\exists x Q^{\prime} M$.

Now let suppose that $Q=\forall x Q^{\prime}$.

If

$$
\text { search_certif_qbf }\left(Q^{\prime},[x \leftarrow \top](M)\right) \text { returns non_valid }
$$

or

$$
\text { search_certif_qbf }\left(Q^{\prime},[x \leftarrow \top](M)\right) \text { returns non_valid }
$$


then

search_certif_qbf $\left(\forall x Q^{\prime}, M\right)$ returns non_valid.

By induction hypothesis one of the two QBF $Q^{\prime}[x \leftarrow \top](M)$ or $Q^{\prime}[x \leftarrow$ $\perp](M)$ is not valid then by definition $\forall x Q^{\prime} M$ is not valid. Otherwise, by induction hypothesis $b l^{+}=$search_certif_qbf $\left(Q^{\prime},[x \leftarrow \top](M)\right)$ is a satcertificate for $Q^{\prime}[x \leftarrow \top](M)$ and $b l^{-}=$search_certif_qbf $\left(Q^{\prime},[x \leftarrow \perp](M)\right)$ is a sat-certificate for $Q^{\prime}[x \leftarrow \perp](M)$ then by Theorem $4\left(b l^{+} \circ_{x} b l^{-}\right)$is a sat-certificate for $\forall x Q^{\prime} M$.

We need also two technical lemmas in order to prove Theorem 6 .

\section{Lemma 1.}

$$
\begin{aligned}
& \left(\left(A \vee\left(\left(P_{1}, N_{1}\right) ; \ldots ;\left(P_{n}, N_{n}\right)\right)^{*}\right) \wedge\left(B \vee\left(\left(P_{1}, N_{1}\right) ; \ldots ;\left(P_{n}, N_{n}\right)\right)^{*}\right)\right) \\
& \equiv\left(\left(\left(P_{1} \vee(A \wedge B)\right),\left(N_{1} \vee(A \wedge B)\right)\right) ; \ldots ;\left(\left(P_{n} \vee(A \wedge B)\right),\left(N_{n} \vee(A \wedge B)\right)\right)\right)^{*}
\end{aligned}
$$

Proof of Lemma 1.

$$
\begin{aligned}
& \left(\left(A \vee\left(\left(P_{1}, N_{1}\right) ; \ldots ;\left(P_{n}, N_{n}\right)\right)^{*}\right) \wedge\left(B \vee\left(\left(P_{1}, N_{1}\right) ; \ldots ;\left(P_{n}, N_{n}\right)\right)^{*}\right)\right) \\
& \equiv\left(\left(A \vee \bigwedge_{1 \leq i \leq n}\left(\left(\neg x_{i} \vee P_{i}\right) \wedge\left(x_{i} \vee N_{i}\right)\right)\right) \wedge\left(B \vee \bigwedge_{1 \leq i \leq n}\left(\left(\neg x_{i} \vee P_{i}\right) \wedge\left(x_{i} \vee N_{i}\right)\right)\right)\right) \\
& \equiv\left((A \wedge B) \vee \bigwedge_{1 \leq i \leq n}\left(\left(\neg x_{i} \vee P_{i}\right) \wedge\left(x_{i} \vee N_{i}\right)\right)\right) \\
& \equiv \bigwedge_{1 \leq i \leq n}\left(\left(\left(\neg x_{i} \vee P_{i}\right) \vee(A \wedge B)\right) \wedge\left(\left(x_{i} \vee N_{i}\right) \vee(A \wedge B)\right)\right) \\
& \equiv \bigwedge_{1 \leq i \leq n}\left(\left(\neg x_{i} \vee\left(P_{i} \vee(A \wedge B)\right)\right) \wedge\left(x_{i} \vee\left(N_{i} \vee(A \wedge B)\right)\right)\right) \\
& \equiv\left(\left(\left(\bar{P}_{1} \vee(A \wedge B)\right),\left(N_{1} \vee(A \wedge B)\right)\right) ; \ldots ;\left(\left(P_{n} \vee(A \wedge B)\right),\left(N_{n} \vee(A \wedge B)\right)\right)\right)^{*}
\end{aligned}
$$

\section{Lemma 2.}

$$
\begin{aligned}
& \left(\left(\left(P_{1}, N_{1}\right) ; \ldots ;\left(P_{n}, N_{n}\right)\right)^{*} \wedge\left(\left(P_{1}^{\prime}, N_{1}^{\prime}\right) ; \ldots ;\left(P_{n}^{\prime}, N_{n}^{\prime}\right)\right)^{*}\right) \\
& \equiv\left(\left(\left(P_{1} \wedge P_{1}^{\prime}\right),\left(N_{1} \wedge N_{1}^{\prime}\right)\right) ; \ldots ;\left(\left(P_{n} \wedge P_{n}^{\prime}\right),\left(N_{n} \wedge N_{n}^{\prime}\right)\right)\right)^{*}
\end{aligned}
$$

Proof of Lemma 2.

$$
\begin{aligned}
& \left(\left(\left(P_{1}, N_{1}\right) ; \ldots ;\left(P_{n}, N_{n}\right)\right)^{*} \wedge\left(\left(P_{1}^{\prime}, N_{1}^{\prime}\right) ; \ldots ;\left(P_{n}^{\prime}, N_{n}^{\prime}\right)\right)^{*}\right) \\
& \equiv\left(\bigwedge_{1 \leq i \leq n}\left(\left(\neg x_{i} \vee P_{i}\right) \wedge\left(x_{i} \vee N_{i}\right)\right) \wedge \bigwedge_{1 \leq i \leq n}\left(\left(\neg x_{i} \vee P_{i}^{\prime}\right) \wedge\left(x_{i} \vee N_{i}^{\prime}\right)\right)\right) \\
& \equiv \bigwedge_{1 \leq i \leq n}\left(\left(\left(\neg x_{i} \vee P_{i}\right) \wedge\left(x_{i} \vee N_{i}\right)\right) \wedge\left(\left(\neg x_{i} \vee P_{i}^{\prime}\right) \wedge\left(x_{i} \vee N_{i}^{\prime}\right)\right)\right) \\
& \equiv \bigwedge_{1 \leq i \leq n}\left(\left(\left(\neg x_{i} \vee P_{i}\right) \wedge\left(\neg x_{i} \vee P_{i}^{\prime}\right)\right) \wedge\left(\left(x_{i} \vee N_{i}\right) \wedge\left(x_{i} \vee N_{i}^{\prime}\right)\right)\right) \\
& \equiv \bigwedge_{1 \leq i \leq n}\left(\left(\neg x_{i} \vee\left(P_{i} \wedge P_{i}^{\prime}\right)\right) \wedge\left(x_{i} \vee\left(N_{i} \wedge N_{i}^{\prime}\right)\right)\right) \\
& \equiv\left(\left(\left(\bar{P}_{1} \wedge P_{1}^{\prime}\right),\left(N_{1} \wedge N_{1}^{\prime}\right)\right) ; \ldots ;\left(\left(P_{n} \wedge P_{n}^{\prime}\right),\left(N_{n} \wedge N_{n}^{\prime}\right)\right)\right)^{*}
\end{aligned}
$$

Theorem 6 Let $Q$ be a binder and $l b, l b^{\prime} \in \mathcal{B}_{Q}$ such that $l b^{*}=Q M$ and $l b^{*}=$ $Q M^{\prime}$. Then $\left(l b \oplus l b^{\prime}\right)^{*}=Q M_{\oplus}$ with $M_{\oplus} \equiv\left(M \vee M^{\prime}\right)$.

Proof of Theorem 6. The theorem holds by definition for the case of an empty binder. Theorem 6 is a direct consequence of the following lemma: Let $Q$ be a non empty binder and $B, B^{\prime} \in \mathcal{B}_{Q}$ such that $B=(Q, G)$ and $B^{\prime}=\left(Q, G^{\prime}\right)$ then $\left(G \oplus G^{\prime}\right)^{*} \equiv\left(G^{*} \vee G^{*}\right)$.

The proof of this lemma is by induction. 
- Base case: $Q=q_{1} x_{1}$.

Let $B=\left(q_{1} x_{1},\left(P_{1}, N_{1}\right)\right)$ and $B^{\prime}=\left(q_{1} x_{1},\left(P_{1}^{\prime}, N_{1}^{\prime}\right)\right)$. Then

$$
\begin{aligned}
& \left(\left(P_{1}, N_{1}\right)^{*} \vee\left(P_{1}^{\prime}, N_{1}^{\prime}\right)^{*}\right) \\
& =\left(\left(\left(\neg x_{1} \vee P_{1}\right) \wedge\left(x_{1} \vee N_{1}\right)\right) \vee\left(\left(\neg x_{1} \vee P_{1}^{\prime}\right) \wedge\left(x_{1} \vee N_{1}^{\prime}\right)\right)\right) \\
& \equiv\left(\left(\neg x_{1} \vee\left(P_{1} \vee P_{1}^{\prime}\right)\right) \wedge\left(x_{1} \vee\left(N_{1} \vee N_{1}^{\prime}\right)\right)\right)
\end{aligned}
$$

By definition $\left(\left(P_{1}, N_{1}\right) \oplus\left(P_{1}^{\prime}, N_{1}^{\prime}\right)\right)=\left(\left(P_{1} \vee P_{1}^{\prime}\right),\left(N_{1} \vee N_{1}^{\prime}\right)\right)$ then

$$
\begin{aligned}
& \left(\left(P_{1}, N_{1}\right) \oplus\left(P_{1}^{\prime}, N_{1}^{\prime}\right)\right)^{*} \\
& =\left(\left(\neg x_{1} \vee\left(P_{1} \vee P_{1}^{\prime}\right)\right) \wedge\left(x_{1} \vee\left(N_{1} \vee N_{1}^{\prime}\right)\right)\right) \\
& =\left(\left(P_{1}, N_{1}\right)^{*} \vee\left(P_{1}^{\prime}, N_{1}^{\prime}\right)^{*}\right)
\end{aligned}
$$

- Induction case: $Q=q_{1} x_{1} \ldots q_{n} x_{n}$.

Let

$$
B=\left(q_{1} x_{1} \ldots q_{n} x_{n},\left(P_{1}, N_{1}\right) ; \ldots ;\left(P_{n}, N_{n}\right)\right)
$$

and

$$
B^{\prime}=\left(q_{1} x_{1} \ldots q_{n} x_{n},\left(P_{1}^{\prime}, N_{1}^{\prime}\right) ; \ldots ;\left(P_{n}^{\prime}, N_{n}^{\prime}\right)\right)
$$

Then by Definition 2

$$
\begin{aligned}
& \left(\left(P_{1}, N_{1}\right) \ldots\left(P_{n}, N_{n}\right)\right)^{*} \\
& =\bigwedge_{1 \leq k \leq n}\left(\left(\neg x_{k} \vee P_{k}\right) \wedge\left(x_{k} \vee N_{k}\right)\right) \\
& =\left(\left(\neg x_{1} \vee P_{1}\right) \wedge\left(x_{1} \vee N_{1}\right)\right) \wedge\left[\left(P_{2}, N_{2}\right) ; \ldots ;\left(P_{n}, N_{n}\right)\right]^{*}
\end{aligned}
$$

and

$$
\begin{aligned}
& \left(\left(P_{1}^{\prime}, N_{1}^{\prime}\right) \ldots\left(P_{n}^{\prime}, N_{n}^{\prime}\right)\right)^{*} \\
& =\bigwedge_{1 \leq k \leq n}\left(\left(\neg x_{k} \vee P_{k}^{\prime}\right) \wedge\left(x_{k} \vee N_{k}^{\prime}\right)\right) \\
& =\left(\left(\neg x_{1} \vee P_{1}^{\prime}\right) \wedge\left(x_{1} \vee N_{1}^{\prime}\right)\right) \wedge\left[\left(P_{2}^{\prime}, N_{2}^{\prime}\right) ; \ldots ;\left(P_{n}^{\prime}, N_{n}^{\prime}\right)\right]^{*}
\end{aligned}
$$

Then from (2) and (3)

$$
\begin{aligned}
( & \left.\left(\left(P_{1}, N_{1}\right) ; \ldots ;\left(P_{n}, N_{n}\right)\right)^{*} \vee\left(\left(P_{1}^{\prime}, N_{1}^{\prime}\right) ; \ldots ;\left(P_{n}^{\prime}, N_{n}^{\prime}\right)\right)^{*}\right) \\
\equiv & \left(\left(\neg x_{1} \vee\left(P_{1} \vee P_{1}^{\prime}\right)\right) \wedge\left(x_{1} \vee\left(N_{1} \vee N_{1}^{\prime}\right)\right)\right) \wedge \\
& \left(\left(\neg x_{1} \vee P_{1}\right) \vee\left[\left(P_{2}^{\prime}, N_{2}^{\prime}\right) ; \ldots ;\left(P_{n}^{\prime}, N_{n}^{\prime}\right)\right]^{*}\right) \wedge \\
& \left(\left(x_{1} \vee N_{1}\right) \vee\left[\left(P_{2}^{\prime}, N_{2}^{\prime}\right) ; \ldots ;\left(P_{n}^{\prime}, N_{n}^{\prime}\right)\right]^{*}\right) \wedge \\
& \left(\left(\neg x_{1} \vee P_{1}^{\prime}\right) \vee\left[\left(P_{2}, N_{2}\right) ; \ldots ;\left(P_{n}, N_{n}\right)\right]^{*}\right) \wedge \\
& \left(\left(x_{1} \vee N_{1}^{\prime}\right) \vee\left[\left(P_{2}, N_{2}\right) ; \ldots ;\left(P_{n}, N_{n}\right)\right]^{*}\right) \wedge \\
& \left(\left[\left(P_{2}, N_{2}\right) ; \ldots ;\left(P_{n}, N_{n}\right)\right]^{*} \vee\left[\left(P_{2}^{\prime}, N_{2}^{\prime}\right) ; \ldots ;\left(P_{n}^{\prime}, N_{n}^{\prime}\right)\right]^{*}\right)
\end{aligned}
$$

Let

$$
\mathcal{X}=\left(\left(\neg x_{1} \vee P_{1}\right) \wedge\left(x_{1} \vee N_{1}\right)\right)
$$

and

$$
\mathcal{X}^{\prime}=\left(\left(\neg x_{1} \vee P_{1}^{\prime}\right) \wedge\left(x_{1} \vee N_{1}^{\prime}\right)\right)
$$

Then from (4), (5), (6) and Lemma 1 


$$
\begin{aligned}
( & \left.\left(\left(P_{1}, N_{1}\right) ; \ldots ;\left(P_{n}, N_{n}\right)\right)^{*} \vee\left(\left(P_{1}^{\prime}, N_{1}^{\prime}\right) ; \ldots ;\left(P_{n}^{\prime}, N_{n}^{\prime}\right)\right)^{*}\right) \\
\equiv & \left(\left(\neg x_{1} \vee\left(P_{1} \vee P_{1}^{\prime}\right)\right) \wedge\left(x_{1} \vee\left(N_{1} \vee N_{1}^{\prime}\right)\right) \wedge\right. \\
& {\left[\left(\left(P_{2}^{\prime} \vee \mathcal{X}\right),\left(N_{2}^{\prime} \vee \mathcal{X}\right)\right) ; \ldots ;\left(\left(P_{n}^{\prime} \vee \mathcal{X}\right),\left(N_{n}^{\prime} \vee \mathcal{X}\right)\right)\right]^{*} \wedge } \\
& {\left[\left(\left(P_{2} \vee \mathcal{X}^{\prime}\right),\left(N_{2} \vee \mathcal{X}^{\prime}\right)\right) ; \ldots ;\left(\left(P_{n} \vee \mathcal{X}^{\prime}\right),\left(N_{n} \vee \mathcal{X}^{\prime}\right)\right)\right]^{*} \wedge } \\
& \left(\left[\left(P_{2}, N_{2}\right) ; \ldots ;\left(P_{n}, N_{n}\right)\right]^{*} \vee\left[\left(P_{2}^{\prime}, N_{2}^{\prime}\right) ; \ldots ;\left(P_{n}^{\prime}, N_{n}^{\prime}\right)\right]^{*}\right)
\end{aligned}
$$

Let

$$
\begin{aligned}
& \left(\mathcal{P}_{2}, \mathcal{N}_{2}\right) ; \ldots ;\left(\mathcal{P}_{n}, \mathcal{N}_{n}\right) \\
& =\left(\left(P_{2}, N_{2}\right) ; \ldots ;\left(P_{n}, N_{n}\right)\right) \oplus\left(\left(P_{2}^{\prime}, N_{2}^{\prime}\right) ; \ldots ;\left(P_{n}^{\prime}, N_{n}^{\prime}\right)\right)
\end{aligned}
$$

By induction hypothesis

$$
\begin{aligned}
& {\left[\left(\mathcal{P}_{2}, \mathcal{N}_{2}\right) ; \ldots ;\left(\mathcal{P}_{n}, \mathcal{N}_{n}\right)\right]^{*}} \\
& \quad=\left(\left[\left(P_{2}, N_{2}\right) ; \ldots ;\left(P_{n}, N_{n}\right)\right]^{*} \vee\left[\left(P_{2}^{\prime}, N_{2}^{\prime}\right) ; \ldots ;\left(P_{n}^{\prime}, N_{n}^{\prime}\right)\right]^{*}\right)
\end{aligned}
$$

Then from (7), (8) and Lemma 2

$$
\begin{aligned}
( & \left.\left(\left(P_{1}, N_{1}\right) ; \ldots ;\left(P_{n}, N_{n}\right)\right)^{*} \vee\left(\left(P_{1}^{\prime}, N_{1}^{\prime}\right) ; \ldots ;\left(P_{n}^{\prime}, N_{n}^{\prime}\right)\right)^{*}\right) \\
\equiv & \left(\left(\neg x_{1} \vee\left(P_{1} \vee P_{1}^{\prime}\right)\right) \wedge\left(x_{1} \vee\left(N_{1} \vee N_{1}^{\prime}\right)\right) \wedge\right. \\
& {\left[\left(\left(P_{2}^{\prime} \vee \mathcal{X}\right),\left(N_{2}^{\prime} \vee \mathcal{X}\right)\right) ; \ldots ;\left(\left(P_{n}^{\prime} \vee \mathcal{X}\right),\left(N_{n}^{\prime} \vee \mathcal{X}\right)\right)\right]^{*} \wedge } \\
& {\left[\left(\left(P_{2} \vee \mathcal{X}^{\prime}\right),\left(N_{2} \vee \mathcal{X}^{\prime}\right)\right) ; \ldots ;\left(\left(P_{n} \vee \mathcal{X}^{\prime}\right),\left(N_{n} \vee \mathcal{X}^{\prime}\right)\right)\right]^{*} \wedge } \\
& {\left[\left(\mathcal{P}_{2}, \mathcal{N}_{2}\right) ; \ldots ;\left(\mathcal{P}_{n}, \mathcal{N}_{n}\right)\right]^{*} } \\
\equiv & \left(\left(\neg x_{1} \vee\left(P_{1} \vee P_{1}^{\prime}\right)\right) \wedge\left(x_{1} \vee\left(N_{1} \vee N_{1}^{\prime}\right)\right)\right) \wedge \\
& {\left[\left(\left(\left(\left(P_{2}^{\prime} \vee \mathcal{X}\right) \wedge\left(P_{2} \vee \mathcal{X}^{\prime}\right)\right) \wedge \mathcal{P}_{2}\right),\left(\left(\left(N_{2}^{\prime} \vee \mathcal{X}\right) \wedge\left(N_{2} \vee \mathcal{X}^{\prime}\right)\right) \wedge \mathcal{N}_{2}\right)\right) ; \ldots ;\right.} \\
& \left.\left(\left(\left(\left(P_{n}^{\prime} \vee \mathcal{X}\right) \wedge\left(P_{n} \vee \mathcal{X}^{\prime}\right)\right) \wedge \mathcal{P}_{n}\right),\left(\left(\left(N_{n}^{\prime} \vee \mathcal{X}\right) \wedge\left(N_{n} \vee \mathcal{X}^{\prime}\right)\right) \wedge \mathcal{N}_{n}\right)\right)\right]^{*} \\
\equiv & \left(\left(\left(P_{1}, N_{1}\right) ; \ldots ;\left(P_{n}, N_{n}\right)\right) \oplus\left(\left(P_{1}^{\prime}, N_{1}^{\prime}\right) ; \ldots ;\left(P_{n}^{\prime}, N_{n}^{\prime}\right)\right)\right)^{*}
\end{aligned}
$$

Theorem 7 Let $Q M$ be a $Q B F$.

search_comp_qbf $(Q, M)$ returns a literal base lb such that $l b^{*} \cong Q M$ if $Q M$ is valid and returns non_valid otherwise.

\section{Proof of Theorem $\%$.}

- Base cases:

- We suppose that $q=\forall$.

* If $M \equiv \top$ then search_comp_qbf $(\forall x, M)=(\forall x(\top, \top))$ then

$$
\text { search_comp_qbf }(\forall x, M)^{*}=\forall x((\neg x \vee \top) \wedge(x \vee \top)) \cong \forall x \top \cong \forall x M
$$

* If $M \equiv x(M \equiv \neg x$ and $M \equiv \perp$ are similar $)$ then $\forall x M$ is not valid and

$$
\text { search_comp_qbf }(\forall x, M)=\text { non_valid }
$$

- We suppose that $q=\exists$.

* If $M \equiv \top$ then

$$
\text { search_comp_qbf }(\exists x, M)=(\exists x(\top, \top))
$$

then

$$
\text { search_comp_qbf }(\exists x, M)^{*}=\exists x((\neg x \vee \top) \wedge(x \vee \top)) \cong \exists x \top \cong \exists x M
$$


* If $M \equiv x$ then

$$
\text { search_comp_qbf }(\exists x, M)=(\exists x(\top, \perp))
$$

then

$$
\text { search_comp_qbf }(\exists x, M)^{*}=\exists x((\neg x \vee \top) \wedge(x \vee \perp)) \cong \exists x x \cong \exists x M
$$

* If $M \equiv \neg x$ then

$$
\text { search_comp_qbf }(\exists x, M)=(\exists x(\perp, \top))
$$

then

$$
\text { search_comp_qbf }(\exists x, M)^{*}=\exists x((\neg x \vee \perp) \wedge(x \vee \top)) \cong \exists x \neg x \cong \exists x M
$$

* If $M \equiv \perp$ then $\exists x M$ is not valid and

$$
\text { search_comp_qbf }(\exists x, M)=\text { non_valid }
$$

- Induction cases: Let $Q=q x Q^{\prime}$ and

$$
b l^{+}=\text {search_comp_qbf }\left(Q^{\prime},[x \leftarrow \top](M)\right)
$$

and

$$
b l^{-}=\text {search_comp_qbf }\left(Q^{\prime},[x \leftarrow \perp](M)\right)
$$

- We suppose that $\left(b l^{+}=n_{0} \_v a l i d\right)$ and $\left(b l^{-}=n_{0} \_v a l i d\right)$. Then by induction hypothesis $Q^{\prime}[x \leftarrow \top](M)$ and $Q^{\prime}[x \leftarrow \perp](M)$ are not valid then $Q M$ is not valid and search_comp_qbf(Q,M) returns non_valid.

- We suppose that $\left(b l^{+} \neq\right.$non_valid $)$and $\left(b l^{-}=\right.$non_valid $)$. By induction hypothesis,

$$
\left(b l^{+}\right)^{*} \cong Q^{\prime}[x \leftarrow \top](M)
$$

If $q=\forall$ it is similar to the case " $\left(b l^{+}=n_{\text {non_valid })}\right.$ and $\left(b l^{-}=\right.$ non_valid)". Otherwise $q=\exists$ and by induction hypothesis

$$
Q^{\prime}[x \leftarrow \perp](M) \text { is not valid. }
$$

Now let

$$
\begin{aligned}
& Q M_{+} \\
& =\left(Q,(\top, \perp) ; \operatorname{grds}\left(b l^{+}\right)\right)^{*} \\
& =\text { search_comp_qbf }(Q, M)^{*}
\end{aligned}
$$

and

$$
Q^{\prime} M^{+}=\left(b l^{+}\right)^{*}
$$

Since by Definition 2

$$
M_{+}=(\neg x \vee \top) \wedge(x \vee \perp) \wedge M^{+}
$$


then

$$
[x \leftarrow \top]\left(M_{+}\right) \equiv M^{+} \text {and }[x \leftarrow \perp]\left(M_{+}\right) \equiv \perp
$$

then

$$
Q^{\prime}[x \leftarrow \top]\left(M_{+}\right) \cong Q^{\prime} M^{+}
$$

and

$$
Q^{\prime}[x \leftarrow \perp]\left(M_{+}\right) \text {is not valid }
$$

then with (5), (4) and (1)

$$
Q^{\prime}[x \leftarrow \top]\left(M_{+}\right) \cong Q^{\prime}[x \leftarrow \top](M)
$$

then with (6) and (2)

$$
Q M_{+} \cong Q M
$$

then with (3)

$$
\text { search_comp_qbf }(Q, M)^{*} \cong Q M .
$$

- We suppose that $\left(b l^{+}=n_{n} \_v a l i d\right)$ and $\left(b l^{-} \neq n o n \_v a l i d\right)$.

The case is similar to the previous case.

- We suppose that $\left(b l^{+} \neq\right.$non_valid $)$and $\left(b l^{-} \neq\right.$non_valid $)$. By induction hypothesis,

$$
\left(b l^{+}\right)^{*} \cong Q^{\prime}[x \leftarrow \top](M)
$$

and

$$
\left(b l^{-}\right)^{*} \cong Q^{\prime}[x \leftarrow \perp](M)
$$

Let

$$
\begin{aligned}
& Q M_{+}=\left(Q,(\top, \perp) ; \operatorname{grds}\left(b l^{+}\right)\right)^{*} \\
& Q M_{-}=\left(Q,(\perp, \top) ; \operatorname{grds}\left(b l^{-}\right)\right)^{*}
\end{aligned}
$$

and

$$
\begin{aligned}
& Q M_{\oplus} \\
& =\left(\left(Q,(\top, \perp) ; \operatorname{grds}\left(b l^{+}\right)\right) \oplus\left(Q,(\perp, \top) ; \operatorname{grds}\left(b l^{-}\right)\right)\right)^{*} \\
& =\text { search_comp_qbf }(Q, M)^{*}
\end{aligned}
$$

By Theorem 6,

$$
M_{\oplus} \equiv\left(M_{+} \vee M_{-}\right)
$$

Let

$$
Q^{\prime} M^{+}=\left(b l^{+}\right)^{*}
$$

and

$$
Q^{\prime} M^{-}=\left(b l^{-}\right)^{*}
$$

By Definition 2, (9) and (13)

$$
M_{+}=(\neg x \vee \top) \wedge(x \vee \perp) \wedge M^{+}
$$

then

$$
[x \leftarrow \top]\left(M_{+}\right) \equiv M^{+}
$$


and

$$
[x \leftarrow \perp]\left(M_{+}\right) \equiv \perp
$$

By Definition 2, (10) and (14)

$$
M_{-}=(\neg x \vee \perp) \wedge(x \vee \top) \wedge M^{-}
$$

then

$$
[x \leftarrow \top]\left(M_{-}\right) \equiv \perp
$$

and

$$
[x \leftarrow \perp]\left(M_{-}\right) \equiv M^{-}
$$

Then from (12), (15) and (17)

$$
[x \leftarrow \top]\left(M_{\oplus}\right) \equiv M^{+}
$$

and from (12), (16) and (18)

$$
[x \leftarrow \perp]\left(M_{\oplus}\right) \equiv M^{-}
$$

Then from (19)

$$
Q^{\prime}[x \leftarrow \top]\left(M_{\oplus}\right) \cong Q^{\prime} M^{+}
$$

and from (20)

$$
Q^{\prime}[x \leftarrow \perp]\left(M_{\oplus}\right) \cong Q^{\prime} M^{-}
$$

Then from (21), (13) and (7)

$$
Q^{\prime}[x \leftarrow \top]\left(M_{\oplus}\right) \cong Q^{\prime}[x \leftarrow \top](M)
$$

and from (22), (14) and (8)

$$
Q^{\prime}[x \leftarrow \perp]\left(M_{\oplus}\right) \cong Q^{\prime}[x \leftarrow \perp](M)
$$

then

$$
Q M_{\oplus} \cong Q M
$$

then from (11)

$$
\text { search_comp_qbf }(Q, M)^{*} \cong Q M .
$$

We need X lemmas in order to prove Theorem 8. In what follows $Q^{i}=$ $q_{1} x_{1} \ldots q_{i} x_{i}$ and $Q_{i}=q_{i} x_{i} \ldots q_{n} x_{n}$.

Lemma 3. Let $Q M$ and $Q M^{\prime}$ be two $Q B F$ such that $Q M \cong Q M^{\prime}, i$ be an integer, $1 \leq i \leq n,\left\{y_{1}, \ldots, y_{p}\right\}$ be the existentially quantified variables of $\left\{x_{1}, \ldots, x_{i-1}\right\}, v_{i-1}=\left[x_{1} \leftarrow C_{1}\right] \ldots\left[x_{i-1} \leftarrow C_{i-1}\right]$ be an interpretation such that there exists a satisfying Skolem function sequence $\hat{y_{1}} ; \ldots ; \hat{y_{p}} ;$ s for $Q^{i-1} Q_{i} M$ and $v_{i-1}$ is in $\hat{y_{1}} ; \ldots ; \hat{y_{p}}$. Then $Q_{i} v_{i-1}(M) \cong Q_{i} v_{i-1}\left(M^{\prime}\right)$.

Proof of Lemma 3. 
- Base case: Obvious for $i=1$.

- Induction case:

- $q_{i}=\exists$. Let $\hat{y_{1}} ; \ldots ; \hat{y_{p}} ; \hat{x_{i}} ; s$ be a satisfying Skolem function sequence for $Q^{i-1} \exists x_{i} Q_{i+1} M$ then by induction hypothesis

$$
\exists x_{i} Q_{i+1} v_{i-1}(M) \cong \exists x_{i} Q_{i+1} v_{i-1}\left(M^{\prime}\right)
$$

We suppose that $\hat{x_{i}}$ for $v_{i-1}$ is equal to true (the proof is similar with false). Then, $v_{i}=\left[x_{1} \leftarrow C_{1}\right] \ldots\left[x_{i-1} \leftarrow C_{i-1}\right]\left[x_{i} \leftarrow \top\right]$ is such that $\hat{y_{1}} ; \ldots ; \hat{y_{p}} ; \hat{x_{i}} ; s$ is a satisfying Skolem function sequence for $Q^{i-1} \exists x_{i} Q_{i+1} M$ and $v_{i}$ is in $\hat{y_{1}} ; \ldots ; \hat{y_{p}} ; \hat{x_{i}}$ and

$$
Q_{i+1} v_{i-1}\left[x_{i} \leftarrow \top\right](M) \cong Q_{i+1} v_{i-1}\left[x_{i} \leftarrow \top\right]\left(M^{\prime}\right)
$$

- $q_{i}=\forall$. Let $\hat{y_{1}} ; \ldots ; \hat{y_{p}} ; s$ be such a satisfying Skolem function sequence for $Q^{i-1} \forall x_{i} Q_{i+1} M$ then by induction hypothesis

$$
\forall x_{i} Q_{i+1} v_{i-1}(M) \cong \forall x_{i} Q_{i+1} v_{i-1}\left(M^{\prime}\right)
$$

Then, $\hat{y_{1}} ; \ldots ; \hat{y_{p}} ; s$ is a satisfying Skolem function sequence for $Q^{i-1} \forall x_{i} Q_{i+1} M$,

$$
Q_{i+1} v_{i-1}\left[x_{i} \leftarrow \top\right](M) \cong Q_{i+1} v_{i-1}\left[x_{i} \leftarrow \top\right]\left(M^{\prime}\right)
$$

and

$$
Q_{i+1} v_{i-1}\left[x_{i} \leftarrow \perp\right](M) \cong Q_{i+1} v_{i-1}\left[x_{i} \leftarrow \perp\right]\left(M^{\prime}\right) .
$$

Lemma 4. Let $Q M$ be a valid $Q B F$ and

$$
\left(Q,\left(P_{1}, N_{1}\right) ; \ldots ;\left(P_{n}, N_{n}\right)\right)=\text { search_comp_qbf }(Q, M) .
$$

Let $i$ be an integer, $1 \leq i \leq n,\left\{y_{1}, \ldots, y_{p}\right\}$ be the existentially quantified variables of $\left\{x_{1}, \ldots, x_{i-1}\right\}$ and $v_{i-1}=\left[x_{1} \leftarrow C_{1}\right] \ldots\left[x_{i-1} \leftarrow C_{i-1}\right]$ be an interpretation such that there exists a satisfying Skolem function sequence $\hat{y_{1}} ; \ldots ; \hat{y_{p}} ; s$ for $Q^{i-1} Q_{i} M$ and $v_{i-1}$ is in $\hat{y_{1}} ; \ldots ; \hat{y_{p}}$ and for all $j, 1 \leq j<i$, if $C_{j}=\top$ then $\models\left[x_{1} \leftarrow C_{1}\right] \ldots\left[x_{j-1} \leftarrow C_{j-1}\right]\left(P_{j}\right)$ else $\models\left[x_{1} \leftarrow C_{1}\right] \ldots\left[x_{j-1} \leftarrow C_{j-1}\right]\left(N_{j}\right)$.

Then

$$
\begin{aligned}
& \text { search_comp_qbf }\left(Q_{i}, v_{i-1}(M)\right)^{*} \\
& \cong Q_{i}\left(\bigwedge_{i \leq k \leq n}\left(\left(\neg x_{k} \vee v_{i-1}\left(P_{k}\right)\right) \wedge\left(x_{k} \vee v_{i-1}\left(N_{k}\right)\right)\right)\right)
\end{aligned}
$$

Proof of Lemma 4.

By Theorem 7

$$
\text { search_comp_qbf } \left.(Q, M)^{*}=Q \bigwedge_{i \leq k \leq n}\left(\left(\neg x_{k} \vee P_{k}\right) \wedge\left(x_{k} \vee N_{k}\right)\right)\right) \cong Q M
$$

Then by Lemma 3

$$
Q_{i} v_{i-1}\left(\bigwedge_{i \leq k \leq n}\left(\left(\neg x_{k} \vee P_{k}\right) \wedge\left(x_{k} \vee N_{k}\right)\right)\right) \cong Q_{i} v_{i-1}(M)
$$


But, by definition of $v_{i-1}$

$$
\begin{aligned}
& v_{i-1}\left(\bigwedge_{1 \leq k \leq n}\left(\left(\neg x_{k} \vee P_{k}\right) \wedge\left(x_{k} \vee N_{k}\right)\right)\right) \\
& \equiv \bigwedge_{i \leq k \leq n}\left(\left(\neg x_{k} \vee v_{i-1}\left(P_{k}\right)\right) \wedge\left(x_{k} \vee v_{i-1}\left(N_{k}\right)\right)\right)
\end{aligned}
$$

Then

$$
\begin{aligned}
& Q_{i} v_{i-1}\left(\bigwedge_{1 \leq k \leq n}\left(\left(\neg x_{k} \vee P_{k}\right) \wedge\left(x_{k} \vee N_{k}\right)\right)\right) \\
& \cong Q_{i} \bigwedge_{i \leq k \leq n}\left(\left(\neg x_{k} \vee v_{i-1}\left(P_{k}\right)\right) \wedge\left(x_{k} \vee v_{i-1}\left(N_{k}\right)\right)\right)
\end{aligned}
$$

From (1) and (2)

$$
\begin{aligned}
& \text { search_comp_qbf }\left(Q_{i}, v_{i-1}(M)\right)^{*} \\
& \cong Q_{1} v_{i-1}(M) \\
& \cong Q_{i} \bigwedge_{i \leq k \leq n}\left(\left(\neg x_{k} \vee v_{i-1}\left(P_{k}\right)\right) \wedge\left(x_{k} \vee v_{i-1}\left(N_{k}\right)\right)\right)
\end{aligned}
$$

Lemma 5. Let $Q M$ be a valid $Q B F$ and

$$
\left(Q,\left(P_{1}, N_{1}\right) ; \ldots ;\left(P_{n}, N_{n}\right)\right)=\text { search_comp_qbf }(Q, M) .
$$

Let $i$ be an integer, $1 \leq i \leq n,\left\{y_{1}, \ldots, y_{p}\right\}$ be the existentially quantified variables of $\left\{x_{1}, \ldots, x_{i-1}\right\}, v_{i-1}=\left[x_{1} \leftarrow C_{1}\right] \ldots\left[x_{i-1} \leftarrow C_{i-1}\right]$ be an interpretation such that there exists a satisfying Skolem function sequence $\hat{y_{1}} ; \ldots ; \hat{y_{p}} ; s$ for $Q^{i-1} Q_{i} M, v_{i-1}$ is in $\hat{y_{1}} ; \ldots ; \hat{y_{p}}$ and for all $k, 1 \leq k<i$, if $C_{k}=\top$ then $=\left[x_{1} \leftarrow C_{1}\right] \ldots\left[x_{k-1} \leftarrow C_{k-1}\right]\left(P_{k}\right)$ else $\models\left[x_{1} \leftarrow C_{1}\right] \ldots\left[x_{k-1} \leftarrow C_{k-1}\right]\left(N_{k}\right)$. Let

$$
\left(Q_{i},\left(P_{i}^{i}, N_{i}^{i}\right) ; \ldots ;\left(P_{n}^{i}, N_{n}^{i}\right)\right)=\text { search_comp_qbf }\left(Q_{i}, v_{i-1}(M)\right) .
$$

Then for all $j, i \leq j \leq n, v_{i-1}\left(P_{j}\right) \equiv P_{j}^{i}$ and $v_{i-1}\left(N_{j}\right) \equiv N_{j}^{i}$.

Proof of Lemma 5. By Lemma 4,

$$
\begin{aligned}
& \text { search_comp_qbf }\left(Q_{i}, v_{i-1}(M)\right)^{*} \\
& \cong Q_{i}\left(\bigwedge_{i \leq k \leq n}\left(\left(\neg x_{k} \vee v_{i-1}\left(P_{k}\right)\right) \wedge\left(x_{k} \vee v_{i-1}\left(N_{k}\right)\right)\right)\right)
\end{aligned}
$$

By Theorem 7,

$$
\begin{aligned}
& \text { search_comp_qbf }\left(Q_{i}, v_{i-1}(M)\right)^{*} \\
& =Q_{i}\left(\bigwedge_{i \leq k \leq n}\left(\left(\neg x_{k} \vee P_{k}^{i}\right) \wedge\left(x_{k} \vee N_{k}^{i}\right)\right)\right)
\end{aligned}
$$

Then, by induction, for all $j, i \leq j \leq n, v_{i-1}\left(P_{j}\right) \equiv P_{j}^{i}$ and $v_{i-1}\left(N_{j}\right) \equiv N_{j}^{i}$.

Theorem 8 Let $Q M$ be a valid $Q B F$. Then search_comp_qbf $(Q, M)$ is optimal. 
Proof of Theorem 8. We have to prove: Let $Q_{1} M$ be a QBF and

$$
\left(Q_{1},\left(P_{1}, N_{1}\right) ; \ldots ;\left(P_{n}, N_{n}\right)\right)=\text { search_comp_qbf }\left(Q_{1}, M\right) .
$$

For all $i, 1 \leq i \leq n$, let $v_{i-1}=\left[x_{1} \leftarrow C_{1}\right] \ldots\left[x_{i-1} \leftarrow C_{i-1}\right]$ be an interpretation such that for all $k, 1 \leq k<i$ if $C_{k}=\top$ then $\models\left[x_{1} \leftarrow C_{1}\right] \ldots\left[x_{k-1} \leftarrow C_{k-1}\right]\left(P_{k}\right)$ else $=\left[x_{1} \leftarrow C_{1}\right] \ldots\left[x_{k-1} \leftarrow C_{k-1}\right]\left(N_{k}\right)$.

Then

$\models v_{i-1}\left(P_{i}\right)$

if and only if there exists a sequence of satisfying Skolem functions for

$Q_{i+1} v_{i-1}\left[x_{i} \leftarrow \top\right]\left(\bigwedge_{1 \leq k \leq n}\left(\left(\neg x_{k} \vee P_{k}\right) \wedge\left(x_{k} \vee N_{k}\right)\right)\right)$

and

$\models v_{i-1}\left(N_{i}\right)$

if and only if there exists a sequence of satisfying Skolem functions for $Q_{i+1} v_{i-1}\left[x_{i} \leftarrow \perp\right]\left(\bigwedge_{1 \leq k \leq n}\left(\left(\neg x_{k} \vee P_{k}\right) \wedge\left(x_{k} \vee N_{k}\right)\right)\right)$.

By the properties of $v_{i-1}$, these consequences are equivalent to:

$=v_{i-1}\left(P_{i}\right)$

if and only if there exists a sequence of satisfying Skolem functions for $Q_{i+1} \bigwedge_{i \leq k \leq n}\left(\left(\neg x_{k} \vee v_{i-1}\left[x_{i} \leftarrow \top\right]\left(P_{k}\right)\right) \wedge\left(x_{k} \vee v_{i-1}\left[x_{i} \leftarrow \top\right]\left(N_{k}\right)\right)\right)$

and

$=v_{i-1}\left(N_{i}\right)$

if and only if there exists a sequence of satisfying Skolem functions for $Q_{i+1} \bigwedge_{i \leq k \leq n}\left(\left(\neg x_{k} \vee v_{i-1}\left[x_{i} \leftarrow \perp\right]\left(P_{k}\right)\right) \wedge\left(x_{k} \vee v_{i-1}\left[x_{i} \leftarrow \perp\right]\left(N_{k}\right)\right)\right)$.

And, by Lemma 4, these consequences are equivalent to

$\models v_{i-1}\left(P_{i}\right)$

if and only if there exists a sequence of satisfying Skolem functions for search_comp_qbf $\left(Q_{i+1}, v_{i-1}\left[x_{i} \leftarrow \top\right](M)\right)$

and

$\models v_{i-1}\left(N_{i}\right)$

if and only if there exists a sequence of satisfying Skolem functions for search_comp_qbf $\left(Q_{i+1}, v_{i-1}\left[x_{i} \leftarrow \perp\right](M)\right)$.

We prove for $\left[x_{i} \leftarrow \perp\right]$, the proof is similar for $T$.

By Theorem 7,

$$
Q_{1} M \cong Q_{1} \bigwedge_{1 \leq j \leq n}\left(\left(\neg x_{j} \vee P_{j}\right) \wedge\left(x_{j} \vee N_{j}\right)\right)
$$

then

$$
Q_{i} v_{i-1}(M) \cong Q_{i} v_{i-1}\left(\bigwedge_{1 \leq j \leq n}\left(\left(\neg x_{j} \vee P_{j}\right) \wedge\left(x_{j} \vee N_{j}\right)\right)\right)
$$


then, by definition of $v_{i-1}$,

$$
\begin{aligned}
& Q_{i} v_{i-1}(M) \\
& \cong Q_{1}\left(\left(\neg x_{i} \vee v_{i-1}\left(P_{i}\right)\right) \wedge\left(x_{i} \vee v_{i-1}\left(N_{i}\right)\right)\right) \\
& \wedge \bigwedge_{i+1 \leq j \leq n}\left(\left(\neg x_{j} \vee v_{i-1}\left(P_{j}\right)\right) \wedge\left(x_{j} \vee v_{i-1}\left(N_{j}\right)\right)\right)
\end{aligned}
$$

then there exists a sequence of satisfying Skolem functions for $Q_{1} v_{i-1}\left[x_{i} \leftarrow\right.$ $\perp](M)$ if and only if there exists a sequence of satisfying Skolem functions for

$$
Q_{i}\left(v_{i-1}\left(N_{i}\right) \wedge \bigwedge_{i+1 \leq j \leq n}\left(\left(\neg x_{j} \vee v_{i-1}\left[x_{i} \leftarrow \perp\right]\left(P_{j}\right)\right) \wedge\left(x_{j} \vee v_{i-1}\left[x_{i} \leftarrow \perp\right]\left(N_{j}\right)\right)\right)\right)
$$

if and only if $\models v_{i-1}\left(N_{i}\right)$ and there exists a sequence of satisfying Skolem functions for

$$
Q_{i+1} \bigwedge_{i+1 \leq j \leq n}\left(\left(\neg x_{j} \vee v_{i-1}\left[x_{i} \leftarrow \perp\right]\left(P_{j}\right)\right) \wedge\left(x_{j} \vee v_{i-1}\left[x_{i} \leftarrow \perp\right]\left(N_{j}\right)\right)\right)
$$

then if $\not \models v_{i-1}\left(N_{i}\right)$ then there is no sequence of satisfying Skolem functions for $Q_{i+1} v_{i-1}\left[x_{i} \leftarrow \perp\right](M)$ then if there exists a sequence of satisfying Skolem functions for $Q_{i+1} v_{i-1}\left[x_{i} \leftarrow \perp\right](M)$ then $\models v_{i-1}\left(N_{i}\right)$.

Now if there is no sequence of satisfying Skolem functions for $Q_{i+1} v_{i-1}\left[x_{i} \leftarrow\right.$ $\perp](M)$ then, by Lemma 5 and definition of the algorithm search_comp_qbf, $v_{i-1}\left(N_{i}\right)=\perp$.

Theorem 9 Let $Q M$ be a valid $Q B F$. Then search_comp_qbf $(Q, M)^{*}$ is minimal.

Proof of Theorem ??.

- Base cases:

- We suppose that $q=\forall$.

* If $M \equiv \top$ then search_comp_qbf $(\forall x, M)=(\forall x(\top, \top))$ then

$$
\text { search_comp_qbf }(\forall x, M)^{*}=\forall x((\neg x \vee \top) \wedge(x \vee \top)) \cong \forall x \top
$$

is minimal.

* If $M \equiv x(M \equiv \neg x$ and $M \equiv \perp$ are similar $)$ then $\forall x M$ is not valid.

- We suppose that $q=\exists$.

* If $M \equiv \top$ then

$$
\text { search_comp_qbf }(\exists x, M)=(\exists x(\top, \top))
$$

then

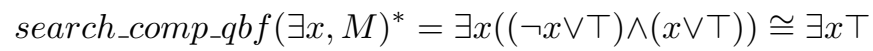

is minimal. 
* If $M \equiv x$ then

$$
\text { search_comp_qbf }(\exists x, M)=(\exists x(\top, \perp))
$$

then

$$
\text { search_comp_qbf }(\exists x, M)^{*}=\exists x((\neg x \vee \top) \wedge(x \vee \perp)) \cong \exists x x
$$

is minimal.

* If $M \equiv \neg x$ then

$$
\text { search_comp_qbf }(\exists x, M)=(\exists x(\perp, \top))
$$

then

$$
\text { search_comp_qbf( }(\exists x, M)^{*}=\exists x((\neg x \vee \perp) \wedge(x \vee \top)) \cong \exists x \neg x
$$

is minimal.

* If $M \equiv \perp$ then $\exists x M$ is not valid.

- Induction cases: Let $Q=q x Q^{\prime}$ and

$$
b l^{+}=\text {search_comp_qbf }\left(Q^{\prime},[x \leftarrow \top](M)\right)
$$

and

$$
b l^{-}=\text {search_comp_qbf }\left(Q^{\prime},[x \leftarrow \perp](M)\right)
$$

- We suppose that $\left(b l^{+}=\right.$non_valid $)$and $\left(b l^{-}=\right.$non_valid $)$. Then by induction hypothesis $Q^{\prime}[x \leftarrow \top](M)$ and $Q^{\prime}[x \leftarrow \perp](M)$ are not valid then $Q M$ is not valid.

- We suppose that $\left(b l^{+} \neq\right.$non_valid $)$and $\left(b l^{-}=\right.$non_valid $)$. By induction hypothesis,

$$
\left(b l^{+}\right)^{*} \text { is minimal. }
$$

If $q=\forall$ it is similar to the case " $\left(b l^{+}=\right.$non_valid $)$and $\left(b l^{-}=\right.$ non_valid)". Otherwise $q=\exists$ and by Theorem 7

$$
Q^{\prime}[x \leftarrow \perp](M) \text { is not valid. }
$$

Now let

$$
\begin{aligned}
& Q M_{+} \\
& =\left(Q,(\top, \perp) ; \operatorname{grds}\left(b l^{+}\right)\right)^{*} \\
& =\text { search_comp_qbf }(Q, M)^{*}
\end{aligned}
$$

and

$$
Q^{\prime} M^{+}=\left(b l^{+}\right)^{*}
$$

Since by Definition 2

$$
M_{+}=(\neg x \vee \top) \wedge(x \vee \perp) \wedge M^{+}
$$

then

$$
[x \leftarrow \top]\left(M_{+}\right) \equiv M^{+} \text {and }[x \leftarrow \perp]\left(M_{+}\right) \equiv \perp
$$


then

$$
Q^{\prime}[x \leftarrow \top]\left(M_{+}\right) \text {is minimal if and only if } Q^{\prime} M^{+} \text {is also minimal }
$$

and

$$
Q^{\prime}[x \leftarrow \perp]\left(M_{+}\right) \text {is not valid. }
$$

then from (5), (4) and (1) $Q^{\prime}[x \leftarrow \top]\left(M_{+}\right)$is minimal then with (6) and (2) $Q M_{+}$is minimal then with (3) search_comp_qbf $(Q, M)^{*}$ is minimal.

- We suppose that $\left(b l^{+}=\right.$non_valid $)$and $\left(b l^{-} \neq\right.$non_valid $)$.

The case is similar to the previous case.

- We suppose that $\left(b l^{+} \neq\right.$non_valid $)$and $\left(b l^{-} \neq\right.$non_valid $)$. By induction hypothesis,

$$
\left(b l^{+}\right)^{*} \text { is minimal }
$$

and

$$
\left(b l^{-}\right)^{*} \text { is minimal. }
$$

Let

$$
\begin{aligned}
& Q M_{+}=\left(Q,(\top, \perp) ; \operatorname{grds}\left(b l^{+}\right)\right)^{*} \\
& Q M_{-}=\left(Q,(\perp, \top) ; \operatorname{grds}\left(b l^{-}\right)\right)^{*}
\end{aligned}
$$

and

$$
\begin{aligned}
& Q M_{\oplus} \\
& =\left(\left(Q,(\top, \perp) ; \operatorname{grds}\left(b l^{+}\right)\right) \oplus\left(Q,(\perp, \top) ; \operatorname{grds}\left(b l^{-}\right)\right)\right)^{*} \\
& =\text { search_comp_qbf }(Q, M)^{*}
\end{aligned}
$$

By Theorem 6,

$$
M_{\oplus} \equiv\left(M_{+} \vee M_{-}\right)
$$

Let

$$
Q^{\prime} M^{+}=\left(b l^{+}\right)^{*}
$$

and

$$
Q^{\prime} M^{-}=\left(b l^{-}\right)^{*}
$$

By Definition 2, (9) and (13)

$$
M_{+}=(\neg x \vee \top) \wedge(x \vee \perp) \wedge M^{+}
$$

then

$$
[x \leftarrow \top]\left(M_{+}\right) \equiv M^{+}
$$

and

$$
[x \leftarrow \perp]\left(M_{+}\right) \equiv \perp
$$

By Definition 2, (10) and (14)

$$
M_{-}=(\neg x \vee \perp) \wedge(x \vee \top) \wedge M^{-}
$$

then

$$
[x \leftarrow \top]\left(M_{-}\right) \equiv \perp
$$


and

$$
[x \leftarrow \perp]\left(M_{-}\right) \equiv M^{-}
$$

Then from (12), (15) and (17)

$$
[x \leftarrow \top]\left(M_{\oplus}\right) \equiv M^{+}
$$

and from (12), (16) and (18)

$$
[x \leftarrow \perp]\left(M_{\oplus}\right) \equiv M^{-}
$$

Then from (19)

$$
Q^{\prime}[x \leftarrow \top]\left(M_{\oplus}\right) \text { is minimal if and only if } Q^{\prime} M^{+} \text {is minimal }
$$

and from (20)

$$
Q^{\prime}[x \leftarrow \perp]\left(M_{\oplus}\right) \text { is minimal if and only if } Q^{\prime} M^{-} \text {is minimal. }
$$

Then from (21), (13) and (7) $Q^{\prime}[x \leftarrow \top]\left(M_{\oplus}\right)$ is minimal and from (22), (14) and (8) $Q^{\prime}[x \leftarrow \perp]\left(M_{\oplus}\right)$ is also minimal then $Q M_{\oplus}$ is minimal then from (11) search_comp_qbf $(Q, M)^{*} \cong Q M$ is minimal. 